\title{
Rapid X-Ray Variations of the Geminga Pulsar Wind Nebula
}

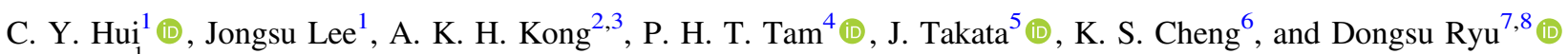 \\ ${ }^{1}$ Department of Astronomy and Space Science, Chungnam National University, Daejeon 34134, Korea; cyhui@cnu.ac.kr \\ ${ }^{2}$ Institute of Astronomy and Department of Physics, National Tsing Hua University, Hsinchu, Taiwan \\ ${ }^{3}$ Astrophysics, Department of Physics, University of Oxford, Keble Road, Oxford OX1 3RH, UK \\ ${ }^{4}$ School of Physics \& Astronomy, Sun Yat-Sen University, Guangzhou 510275, China \\ ${ }^{5}$ Institute of Particle Physics and Astronomy, Huazhong University of Science and Technology, Wuhan, China \\ ${ }^{6}$ Department of Physics, University of Hong Kong, Pokfulam Road, Hong Kong \\ ${ }^{7}$ Department of Physics, UNIST, Ulsan 44919, Korea \\ ${ }^{8}$ Korea Astronomy and Space Science Institute, Daejeon 34055, Korea \\ Received 2017 March 31; revised 2017 August 4; accepted 2017 August 9; published 2017 September 8
}

\begin{abstract}
A recent study by Posselt et al. reported the deepest X-ray investigation of the Geminga pulsar wind nebula (PWN) by using Chandra X-ray Observatory. In comparison with previous studies of this system, a number of new findings have been reported, and we found that these suggest the possible variabilities in various components of this PWN. This motivates us to carry out a dedicated search for the morphological and spectral variations of this complex nebula. We have discovered variabilities on timescales from a few days to a few months from different components of the nebula. The fastest change occurred in the circumstellar environment at a rate of $80 \%$ of the speed of light. One of the most spectacular results is the wiggling of a half light-year long tail as an extension of the jet, which is significantly bent by the ram pressure. The jet wiggling occurred at a rate of about $20 \%$ of the speed of light. This twisted structure could possibly be a result of a propagating torsional Alfv́en wave. We have also found evidence of spectral hardening along this tail for a period of about nine months.
\end{abstract}

Key words: stars: pulsar: individual (PSR J0633+1746, Geminga)

Supporting material: animation, interactive figures

\section{Introduction}

It is generally believed that most of the rotational energy from a pulsar is carried away by a relativistic particle outflow known as pulsar wind (Gold 1969). When these fast-moving wind particles interact with the interstellar medium, shocked emission can be formed, leading to the formation of pulsar wind nebulae (PWNe) (Rees \& Gunn 1974; Arons 2012). Theoretical models suggest that the morphology of PWNe is manipulated by magnetohydrodynamic (MHD) instability of the outflows (Mizuno et al. 2014; Singh et al. 2016). Therefore, studying the variabilities of $\mathrm{PWNe}$ provides a means to investigate the underlying MHD activities.

Geminga (PSR J0633+1746) is a nearby $(d \sim 250 \mathrm{pc})$, middle-aged $\left(\tau=3.4 \times 10^{5} \mathrm{yr}\right)$, radio-quiet $\gamma$-ray pulsar with a spin-down power of $\dot{E}=3.3 \times 10^{34} \mathrm{erg} \mathrm{s}^{-1}$ (Manchester et al. 2005; Faherty et al. 2007). Its projected velocity of $v \sim 211 \mathrm{~km} \mathrm{~s}^{-1}$ suggests that Geminga is in a supersonic motion and should be associated with a bow shock PWN. Given its proximity, it is expected to provide an ideal system for resolving and investigating various components of the shock emission.

With the first Chandra observation of Geminga in 2004, Sanwal et al. (2004) and Pavlov et al. (2006) found an axial tail that extends at least up to $\sim 25^{\prime \prime}$ directly behind Geminga's proper-motion direction. In revisiting this system with the second Chandra observation in 2007, Pavlov et al. (2010) showed that the axial tail extends for $\sim 50^{\prime \prime}$ from the pulsar. Several blobs have also been found on the axial tail. Comparing the images obtained in 2004 and 2007, Pavlov et al. (2010) noticed the possible variability of Geminga's axial tail in terms of its spectrum and brightness and the appearance of blobs along it.
With this deeper observation, two faint $\sim 2^{\prime}$ long outer tails as first found by Caraveo et al. (2003) with XMM-Newton are also detected. Both tails are significantly bent away from the direction of proper motion.

Very recently, Posselt et al. (2017) reported a detailed study of Geminga's PWN with 12 more new Chandra observations carried out from 2012 November to 2013 September. Based on spatial analysis, they confirmed the morphological variability of the axial tail as reported by Pavlov et al. (2010). However, only the combined spectrum was examined in their study. It remains unknown whether there is any associated spectral variability.

Also, Posselt et al. (2017) found that the combined spectrum of the southern outer tail is significantly harder than that of the axial tail (cf. Table 3 in Posselt et al. 2017). This is different from the results reported by Pavlov et al. (2010), which found that the photon indices of the southern tail and the axial tail are comparable in the earlier observations in 2004 and 2007 (cf. Table 1 in Pavlov et al. 2010). This indicates a possible variability in the southern tail such that its spectrum was hardened in a certain epoch.

Another interesting discovery reported by Posselt et al. (2017) are the new components found in the immediate surroundings of the pulsar (cf. Figure 6 in their paper). The most prominent one is the southeastern feature that connects the pulsar and the southern outer tail. This component can only be found in several epochs. This also suggests the possible variability of this component. However, the significance of the feature is not mentioned in Posselt et al. (2017). It remains unclear if this small-scale feature is genuine or merely a result of background fluctuation. 
Table 1

Details of Chandra Observations of the Field of Geminga in Different Epochs

\begin{tabular}{|c|c|c|c|c|}
\hline Obs. ID. & Detector & $\begin{array}{l}\text { Start Date and Time } \\
\text { (UTC) }\end{array}$ & Mode & $\begin{array}{l}\text { Exposure } \\
(\mathrm{ks})\end{array}$ \\
\hline \multicolumn{5}{|c|}{ Epoch 1} \\
\hline 4674 & ACIS-S & 2004 Feb 07 13:01:59 & FAINT & 18.8 \\
\hline \multicolumn{5}{|c|}{ Epoch 2} \\
\hline 7592 & ACIS-I & 2007 Aug 27 10:10:05 & VFAINT & 77.1 \\
\hline \multicolumn{5}{|c|}{ Epoch 3} \\
\hline 15595 & ACIS-I & 2012 Nov 28 22:18:14 & VFAINT & 62.24 \\
\hline 14691 & ACIS-I & 2012 Dec 01 17:09:00 & VFAINT & 36.58 \\
\hline \multicolumn{5}{|c|}{ Epoch 4} \\
\hline 14692 & ACIS-I & 2013 Jan 25 07:40:57 & VFAINT & 103.68 \\
\hline \multicolumn{5}{|c|}{ Epoch 5} \\
\hline 15623 & ACIS-I & 2013 Mar 19 05:42:00 & VFAINT & 23.75 \\
\hline 15622 & ACIS-I & 2013 Mar 24 12:10:42 & VFAINT & 47.04 \\
\hline 14693 & ACIS-I & 2013 Mar 27 03:49:08 & VFAINT & 22.76 \\
\hline \multicolumn{5}{|c|}{ Epoch 6} \\
\hline 14694 & ACIS-I & 2013 Apr 22 17:37:27 & VFAINT & 96.25 \\
\hline \multicolumn{5}{|c|}{ Epoch 7} \\
\hline 15551 & ACIS-I & 2013 Aug 25 20:12:33 & VFAINT & 30.66 \\
\hline 16318 & ACIS-I & 2013 Aug 28 13:21:30 & VFAINT & 19.81 \\
\hline 16319 & ACIS-I & 2013 Aug 30 12:31:46 & VFAINT & 44.48 \\
\hline \multicolumn{5}{|c|}{ Epoch 8} \\
\hline 15552 & ACIS-I & 2013 Sep 16 03:42:22 & VFAINT & 37.08 \\
\hline 16372 & ACIS-I & 2013 Sep 20 13:17:03 & VFAINT & 59.28 \\
\hline
\end{tabular}

Motivated by all these new findings, we have performed a follow-up investigation of this complex X-ray nebula with an aim to search for the variabilities in different components.

\section{Observation and Data Reduction}

All the data used in this investigation were obtained by the Advanced CCD Imaging Spectrometer (ACIS) onboard Chandra. The details of these observations are summarized in Table 1. For the data reduction, we utilized the Chandra Interactive Analysis of Observations software (CIAO) with the updated calibration database (CALDB). Using the CIAO script chandra_repro, we reprocessed all the data with subpixel event repositioning in order to facilitate a high angular resolution analysis. Since the instrumental background increases sharply below $0.5 \mathrm{keV}$ on the front-illuminated CCDs (ACIS-I) that were used in all observations except the one in 2004, we apply the low-energy cut at $0.5 \mathrm{keV}$. All the investigations in this work were restricted to an energy range of $0.5-7 \mathrm{keV}$.

To begin with, we have performed a preliminary screening of all the full-field images from individual observations by visual inspection. For those observations separated by less than 10 days, we do not find any obvious variability among the images in this preliminary check. In view of this, we group the data into eight different epochs as defined in Table 1 so as to optimize the photon statistic for the subsequent analysis. Hereafter, we adopt the definition of these epochs for referring to the time frame in our analysis. With this scheme of data grouping, the effective exposures from Epoch 3 to Epoch 8 are found to be comparable ( $\sim 100 \mathrm{ks})$. This scheme is consistent with that adopted by Posselt et al. (2017).

In this investigation, we only consider spatial features that are detected at a signal-to-noise ratio $(\mathrm{S} / \mathrm{N})>5 \sigma$ as genuine. This criterion allows us to better discriminate the variable diffuse emission from the background fluctuations.

We followed the method in $\mathrm{Li} \& \mathrm{Ma}$ (1983) in calculating the $\mathrm{S} / \mathrm{Ns}$ for all features in our work. The net source counts $N_{\text {net }}$ in a region of interest of an area $A_{S}$ is estimated by $N_{\text {net }}=N_{\text {tot }}-\alpha N_{\text {bkg }}$, where $N_{\text {tot }}$ is the total counts sampled in $A_{S}$, $N_{\text {bkg }}$ is the background counts sampled in a source-free region of an area $A_{B}$, and $\alpha=A_{S} / A_{B}$. The estimate of the standard deviation of $N_{\text {net }}$ is given by

$$
\hat{\sigma}\left(N_{\text {net }}\right)=\sqrt{N_{\text {net }}+(1+\alpha) \alpha N_{\mathrm{bkg}}} .
$$

Hence, $\mathrm{S} / \mathrm{N}$ is estimated as $N_{\text {net }} / \hat{\sigma}\left(N_{\text {net }}\right)$.

\section{Variability Analysis of Various Spatial Components}

Figure 1 shows the $6^{\prime} \times 6^{\prime}$ field of Geminga and its PWN, which combines all the X-ray observations that cover the entire PWN. Apart from the pulsar as the brightest pointlike source, there are two distinct extended structures near the pulsar: the axial tail and outer tails, as illustrated in Figure 1. The axial tail is intimately connected to the pulsar and spans an apparent length of $\sim 42^{\prime \prime}(0.05 \mathrm{pc})$ from the pulsar position to a bright blob at the end of the feature (i.e., Blob C, as illustrated in Figures 2 and 3 ) in this combined image. Besides this bright blob, the axial tail also appears to comprise several clumpy structures (see Section 3.1 for further details). Besides the axial tail, two outer tails extending out to about $2^{\prime}(0.15 \mathrm{pc})$ can be clearly seen. A detailed analysis of these two long tails will be presented in Section 3.3.

From this energy-coded color image, we notice that the $\mathrm{X}$-rays from both outer tails are harder than those from the axial tail. This is consistent with the results reported by Posselt et al. (2017) based on their analysis of combined spectra. For the southern outer tail, we also note that the X-rays are apparently harder in the region further away from the pulsar.

In the following, we investigate the variability of each component through a detailed spectral-imaging analysis.

\subsection{Axial Tail}

In this work, we define the axial tail as a feature that is intimately connected to the pulsar. Figure 2 clearly shows the variability of the axial tail. All the images in this work are exposure-corrected by using the CIAO script fluximage. We also overlaid the locations of Blobs A, B, and C as found by Pavlov et al. (2010) on all images for comparison.

For quantifying their lengths in each epoch, we computed their brightness profiles by using the unsmoothed image with a bin size of 0 ". $5 \times 0$ ". 5 in a $60^{\prime \prime} \times 10^{\prime \prime}$ region along the tail, which is large enough to cover all the identified features for the axial in different epochs. The results are shown in Figure 3 . The peak of the profiles corresponds to the location of the pulsar. The shift of the peak among these profiles is a result of the proper motion of Geminga. For the background level in each epoch, we randomly sampled in 10 source-free regions of the corresponding image and computed the medium and the $1 \sigma$ uncertainties, illustrated by the solid 


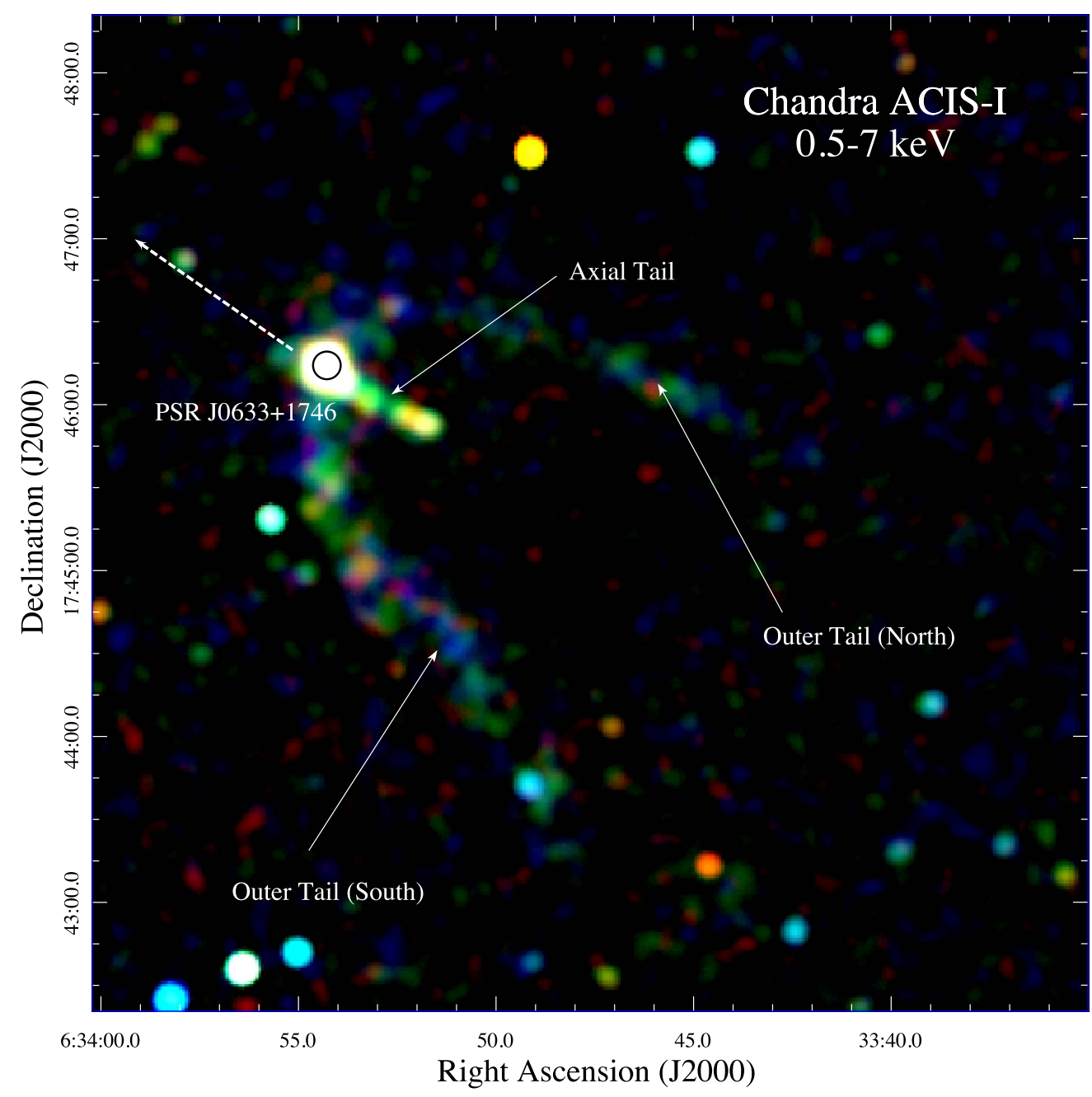

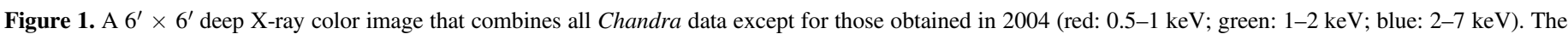

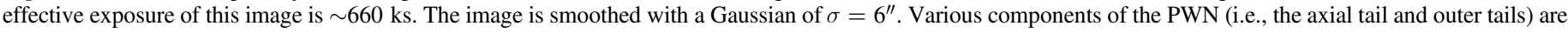
illustrated accordingly. The dashed arrow shows the direction of Geminga's proper motion.

and dashed horizontal lines, respectively, in Figure 3. The length of the axial tail in each epoch is estimated by the profile of the feature extended behind the pulsar before it falls into the background.

The variability of the axial tail and blobs is obvious from Figures 2 and 3. Since the morphological changes of these feature have been studied in detail by Posselt et al. (2017), we do not repeat this analysis. We refer interested readers to Section 2.4 of their paper.

In our work, we focus in examining if there is temporal variation of the spectral properties. We extracted the source spectrum of the axial tail from each observation according to its extent defined by the brightness profile (Figure 3) in the corresponding epoch. For the sake of consistency, we followed the definition that the tail is a feature that is intimately connected to the pulsar. We extracted the spectrum using a single box that covers the feature and excluded those features that apparently disconnected from it. For example, Blob B is included in the data of Epochs 2 and 6 but is excluded in Epoch 4. The background spectra were sampled from the source-free regions in the individual data. We utilized the CIAO tool specextract to extract all the spectra and compute the response files.
For the spectral analysis, we used XSPEC throughout this investigation. For obtaining results that can be compared to those reported by Pavlov et al. (2010) and Posselt et al. (2017), we followed their procedures in the spectral fitting. We fitted all the spectra with an absorbed power-law model with the column absorption fixed at $N_{H}=1.1 \times 10^{20} \mathrm{~cm}^{-2}$ (Halpern \& Wang 1997; De Luca et al. 2005). All the observed fluxes and absorption-corrected luminosities quoted in this work are computed in $0.3-8 \mathrm{keV}$ to make the comparison with the results of Pavlov et al. (2010) and Posselt et al. (2017) easier.

The spectral properties of the axial tail in different epochs are summarized in Table 2. The background-subtracted spectra and fitting residuals are shown in Figure 4. Our results in Epochs 1 and 2 agree with those reported by Pavlov et al. (2010). Comparing the photon indices inferred from various epochs, there is no significant variation within the tolerance of their $1 \sigma$ uncertainties. In Epoch 7, we found that the spectral results obtained individually from the data obtained on 2013 August 25 (ObsID: 15551) and 28 (ObsID: 16318) are consistent. However, such results are not consistent with those obtained on August 30 (ObsID: 16319). We reexamined all spectra from Epoch 7 with backgrounds selected from a set of different nearby source-free regions, and we found that the difference 

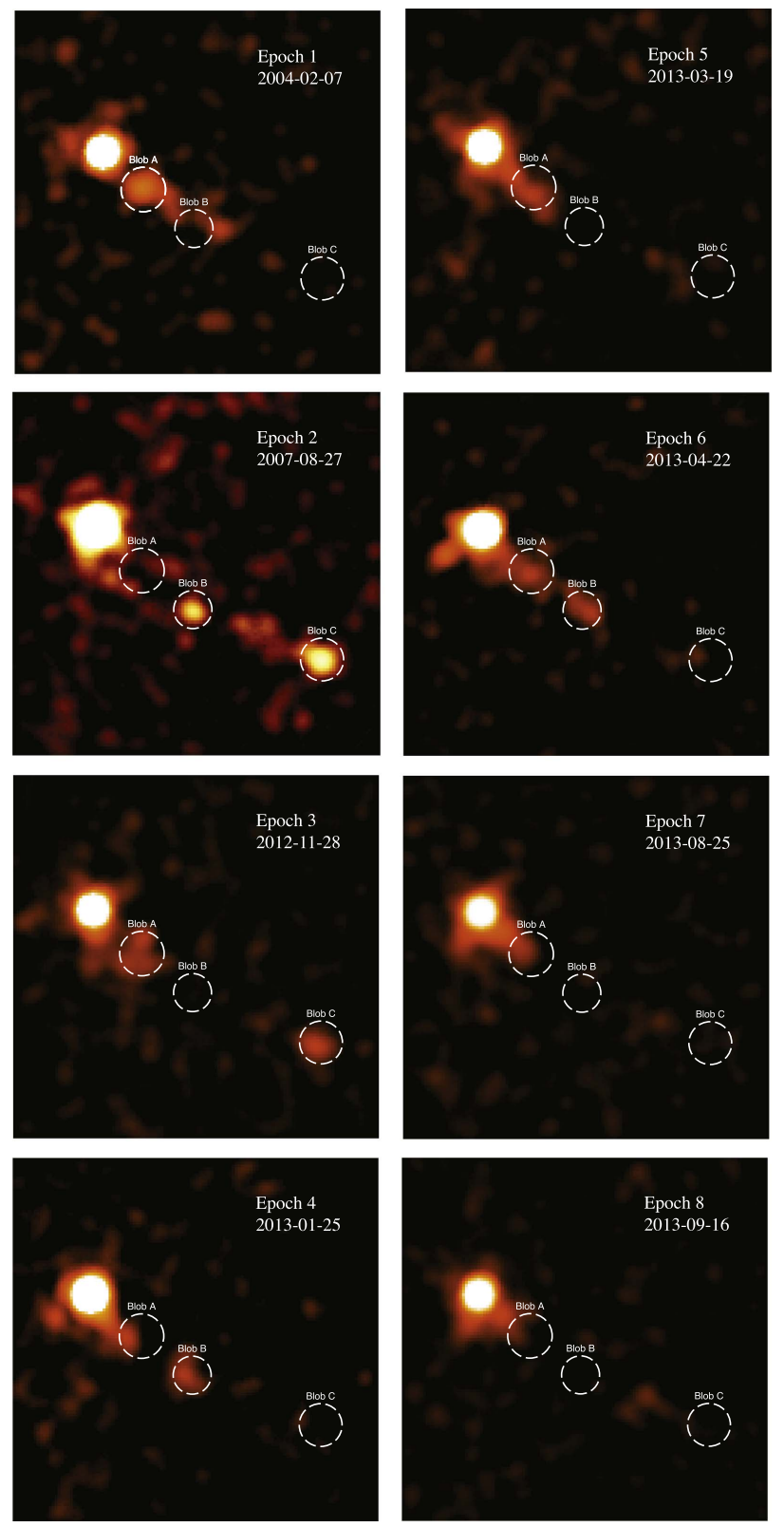

Figure 2. Time sequence of Chandra $1^{\prime} \times 1^{\prime}$ exposure-corrected images $(0.5-7 \mathrm{keV})$ of the field around Geminga's axial tail $(0$ " $5 \times 0$.. 5 for 1 pixel $)$. The start date of the corresponding epoch is given in each image. All images are smoothed with a Gaussian kernel of $\sigma=2$ ". 5 . The dashed circles illustrate the locations of Blobs A, B, and C, detected previously (Pavlov et al. 2010).

(An animation of this figure is available.)

persists. It is not possible to properly characterize the spectral properties of the axial tail in this epoch by combining all data. This leads us to further divide Epoch 7 into two subframes, namely Epoch 7a (ObsID: 15551 + ObsID: 16318) and Epoch 7b (ObsID: 16319). We compare the confidence contours in the parameter space spanned by the photon index and the model normalization for these two subframes in Figure 5. It is interesting to note that the X-ray spectrum of the axial tail can vary on a timescale as short as a few days.

However, we noted that the $2 \sigma$ and $3 \sigma$ contours in these two subframes are overlapped. To further investigate the variability, we examined the individual images in Epochs $7 \mathrm{a}$ and $7 \mathrm{~b}$ separately to see if there was any corresponding morphological variation. We examined the images of these two frames in a $30^{\prime \prime} \times 30^{\prime \prime}$ region centered on the pulsar. To enable a more sensitive search for the morphological variation of a small-scale feature, we produced the images with subpixel resolution with a binning factor of 0.5 . By comparing these two exposurecorrected images, which are both adjusted to the same logarithmic color scale (upper panels of Figure 7), the tail behind proper-motion direction appears to dim in less than five days. Such a result is consistent with that based on the spectral analysis and supports the presence of such short-timescale variability.

In view of the discovery of fast variability in Epoch 7, we have also examined the possible spectral and morphological variabilities of the axial tail among the individual observations in Epochs 3, 5, and 8. However, no short-term variability has been found within these epochs.

We also examined the epoch-averaged spectral properties of the axial tail so as to cross-check the results of Posselt et al. (2017). Instead of fitting the combined spectrum as was done by Posselt et al. (2017), we adopted a more rigorous approach by fitting all 12 individual axial tail spectra simultaneously. We found that the epoch-averaged spectrum can be described by a power law with a photon index of $\Gamma=1.76 \pm 0.17$ and a normalization factor of $(1.6 \pm 0.2) \times 10^{-6}$ photons $\mathrm{keV}^{-1} \mathrm{~cm}^{-2} \mathrm{~s}^{-1}$ at $1 \mathrm{keV}$. Within the tolerance of the statistical uncertainties, the results are consistent with those reported by Posselt et al. (2017; cf. Table 3 in their paper).

\subsection{Circumstellar Environment}

Inspecting Figure 2, we noted the presence of extended emission of a few arcseconds in the vicinity of the pulsar. For example, the protrusions in the directions that connected to the outer tails in Epochs 7 and 8 are particularly prominent. In Section 3.2, we will explore these small-scale features in detail.

We have performed a detailed morphological study of the circumstellar environment to search for the possible diffuse $\mathrm{X}$-ray feature around the pulsar. For each observation, we have produced a $30^{\prime \prime} \times 30^{\prime \prime}$ exposure-corrected image centered at the pulsar with subpixel resolution $(0$ ". $25 \times 0$ ". 25 for 1 pixel $)$. We have identified the possible compact extended features around the pulsar in Epochs 4, 6, 7, and 8 through visual inspections of these images. These extended-feature candidates are shown in Figure 6. All these features resemble a collimated protrusion that extends to the southeast and is connected to the southern outer tail.

Posselt et al. (2017) also spotted this feature in Epochs 4, 6, and 8 (cf. Section 2.3 in their paper). However, the detection significance has not been addressed. We have examined this putative feature with a more detailed analysis. Estimated with the nearby source-free background regions, we have found that the S/Ns of the features identified in Epochs 4 and 6 are only at the level of $\sim 4.5 \sigma$. According to our predefined detection criterion, we are not able to firmly distinguish if they are genuine or the result of background fluctuations. For Epoch 7, we have investigated Epoch 7a and Epoch 7b separately. Their images are shown in the upper panels of Figure 7. A southeastern protrusion is noted in both Epoch 7a and Epoch 7b. The feature can be detected at an $\mathrm{S} / \mathrm{N}$ of $5.1 \sigma$ in both epochs. We have also examined the robustness of these detections with various source-free backgrounds in nearby 

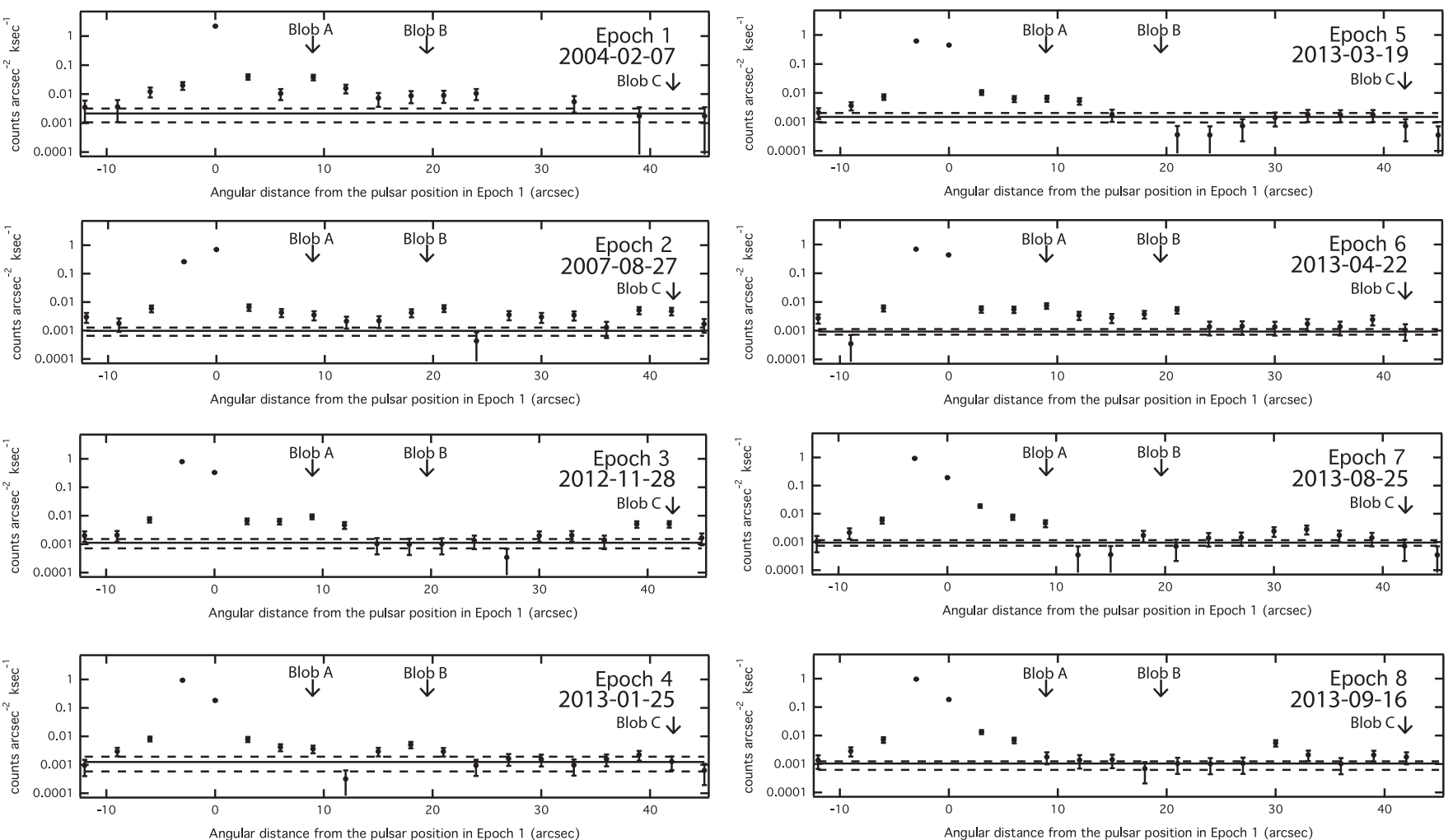

Figure 3. Brightness profiles of the axial tail as computed from a $60^{\prime \prime} \times 10^{\prime \prime}$ region along the feature from the unsmoothed images in different epochs. The opposed direction of the pulsar's proper motion is defined as positive in the $x$ axis. The horizontal solid and dashed lines represent the background and its $1 \sigma$ uncertainties at each epoch. The locations of Blobs A, B, and C as shown in Figure 2 are illustrated by the arrows accordingly.

Table 2

Spectral Properties of the Axial Tail in Different Epochs

\begin{tabular}{|c|c|c|c|c|c|c|c|c|}
\hline & $\begin{array}{c}A_{S} \\
\left(\operatorname{arcsec}^{2}\right)\end{array}$ & $\begin{array}{c}N_{\text {net }} \\
\text { counts }\end{array}$ & $\begin{array}{c}N_{\text {bkg }} \\
\text { counts }\end{array}$ & $\bar{\alpha}$ & $\begin{array}{c}\mathrm{S} / \mathrm{N} \\
(\sigma)\end{array}$ & $\bar{\Gamma}$ & $\begin{array}{c}F_{\mathrm{obs}}{ }^{\mathrm{a}} \\
\left(10^{-14} \mathrm{erg} \mathrm{cm}^{-2} \mathrm{~s}^{-1}\right)\end{array}$ & $\begin{array}{c}L_{\text {unabsorb }}{ }^{\mathrm{a}} \\
\left(10^{29} \mathrm{erg} \mathrm{s}^{-1}\right)\end{array}$ \\
\hline Epoch 1 & 120.8 & 44 & 7 & 0.77 & 6.0 & $1.27_{-0.40}^{+0.46}$ & $2.40_{-0.61}^{+0.74}$ & $1.82_{-0.45}^{+0.55}$ \\
\hline Epoch 2 & 337.9 & 65 & 42 & 0.93 & 5.5 & $1.98_{-0.34}^{+0.40}$ & $1.12_{-0.19}^{+0.20}$ & $0.91_{-0.14}^{+0.15}$ \\
\hline Epoch 3 & 108.9 & 80 & 14 & 1.00 & 7.7 & $1.92_{-0.36}^{+0.43}$ & $1.08 \pm 0.15$ & $0.84 \pm 0.12$ \\
\hline Epoch 4 & 62.7 & 42 & 9 & 1.01 & 5.4 & $1.46_{-0.50}^{+0.54}$ & $0.64_{-0.15}^{+0.21}$ & $0.49_{-0.11}^{+0.16}$ \\
\hline Epoch 5 & 102.1 & 63 & 9 & 1.00 & 7.0 & $1.41_{-0.61}^{+0.57}$ & $1.19_{-0.23}^{+0.24}$ & $0.90_{-0.18}^{+0.20}$ \\
\hline Epoch 7b & 60.5 & 29 & 4 & 0.44 & 5.2 & $1.04_{-0.46}^{+0.48}$ & $1.15_{-0.28}^{+0.40}$ & $0.87_{-0.21}^{+0.29}$ \\
\hline Epoch 8 & 38.03 & 45 & 10 & 0.33 & 6.4 & $1.09_{-0.40}^{+0.44}$ & $0.50_{-0.14}^{+0.15}$ & $0.38 \pm 0.11$ \\
\hline
\end{tabular}

Note.

${ }^{\mathrm{a}}$ The observed fluxes $F_{\mathrm{obs}}$ and the absorption-corrected luminosities $L_{\text {unabsorb }}$ (adopting $d=250 \mathrm{pc}$ ) are computed for the energy range of $0.3-8 \mathrm{keV}$.

regions. We have found that the $\mathrm{S} / \mathrm{Ns}$ of the features remain above our predefined threshold in both epochs.

For quantifying the length of the southeastern protrusion, we computed the brightness profiles from the unsmoothed subpixel-resolution images in Epoch $7 \mathrm{a}$ and $7 \mathrm{~b}$, shown in the upper panels of Figure 8. The length of the feature is estimated by its profile extending in a southeastern direction from the pulsar before it falls into the background. Comparing Epoch 7a and Epoch $7 \mathrm{~b}$, the feature is found to be lengthened by $\sim 2 ! .5$ within $\sim 5$ days. At a distance of $250 \mathrm{pc}$, the change in its physical length is $\sim 9.4 \times 10^{15} \mathrm{~cm}$. This implies that the variability of this feature occurred at a speed of $\sim 0.8 c$. With the column absorption fixed at $1.1 \times 10^{20} \mathrm{~cm}^{-2}$, its spectrum can be described by a power-law model with a photon index of $\Gamma=1.16 \pm 0.48$. Its absorption-corrected flux is at the level of $f_{x} \sim 1.0 \times 10^{-14} \mathrm{erg} \mathrm{cm}^{-2} \mathrm{~s}^{-1}(0.3-8 \mathrm{keV})$.

For Epoch 8, we have also separately analyzed the observations in 2013 September 16 (Epoch 8a) and 20 (Epoch $8 \mathrm{~b})$. The close-up images of the pulsar obtained by these two observations are shown in the lower panels of Figure 7. While the protrusion is undetected in Epoch 8a, it is found at a significance of $5.3 \sigma$ in Epoch $8 \mathrm{~b}$. Comparing the brightness profiles in Epoch $8 \mathrm{a}$ and $8 \mathrm{~b}$ (lower panels of Figure 8) shows that the protrusion was extended by $\sim 2^{\prime \prime}$ in Epoch 8 b. These show 

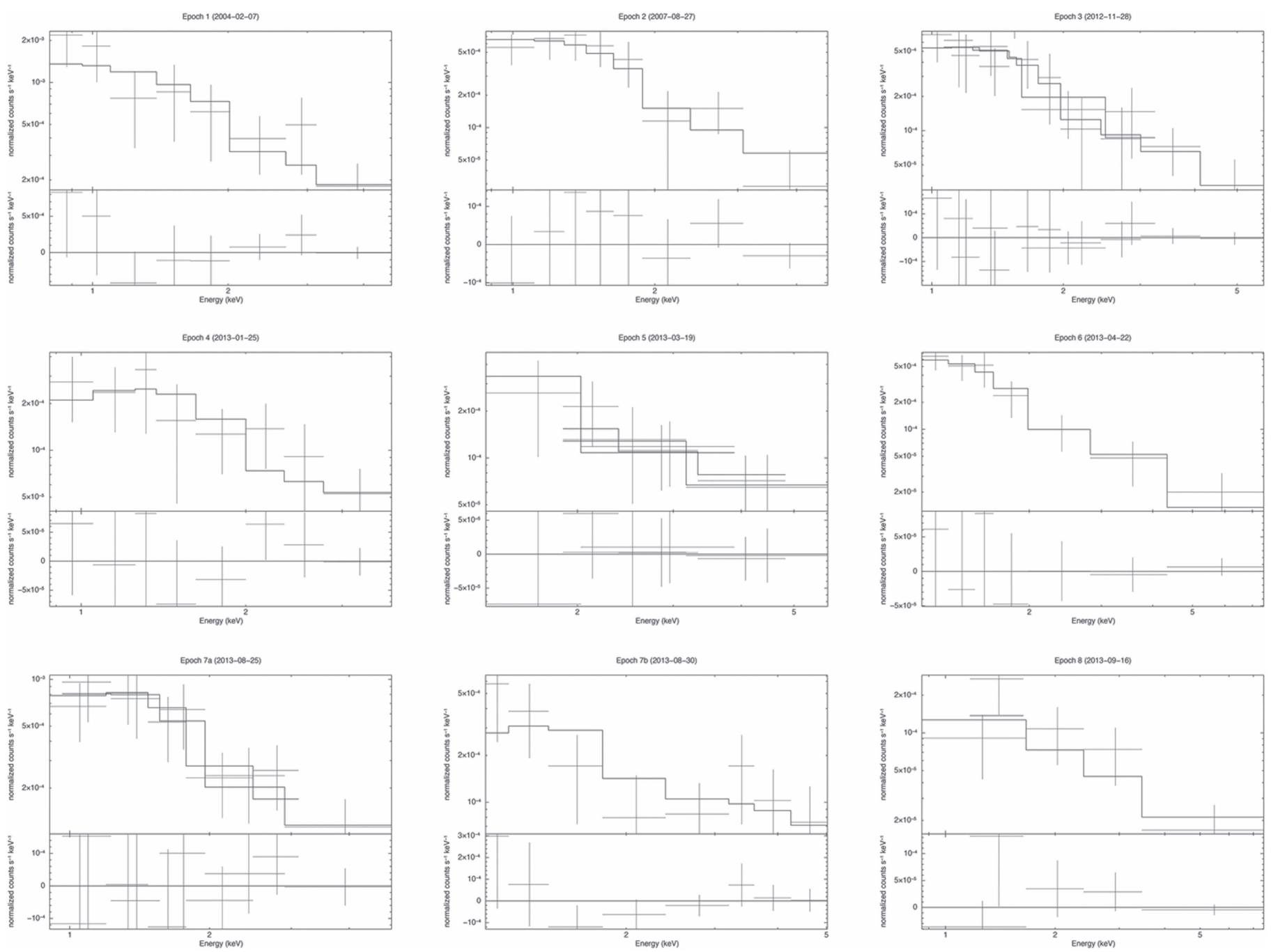

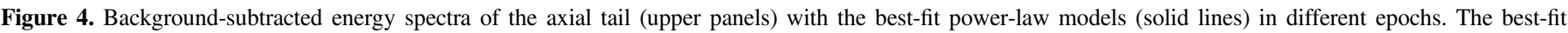
spectral parameters are given in Table 2. The fitting residuals are also shown (lower panels).

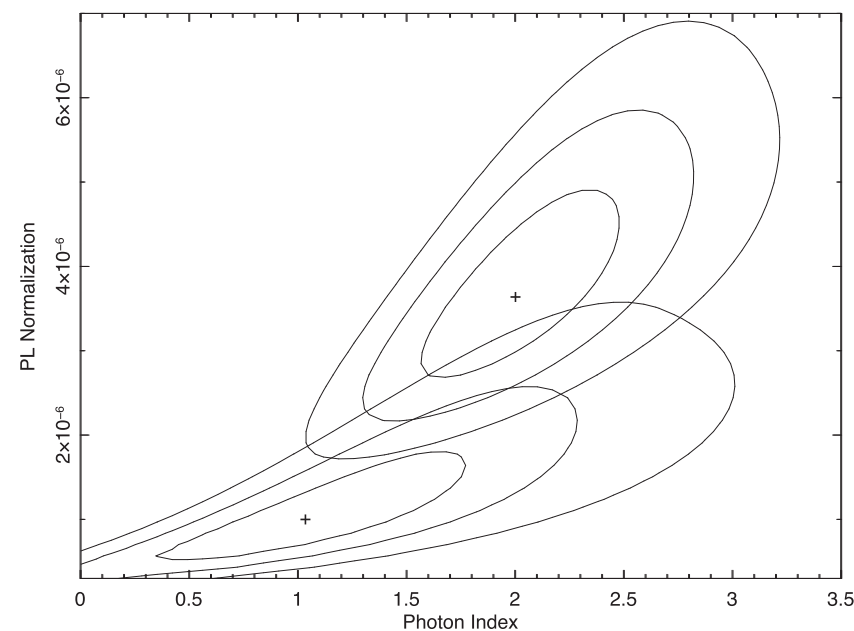

Figure 5. The $1 \sigma, 2 \sigma$, and $3 \sigma$ confidence contours for the absorbed power-law model fitted to the X-ray spectra of Geminga's axial tail as observed by Chandra in Epoch 7a (upper contours) and Epoch 7b (lower contours). The unit of the model normalization is photons $\mathrm{keV}^{-1} \mathrm{~cm}^{-2} \mathrm{~s}^{-1}$ at $1 \mathrm{keV}$.

the rapid variability of this feature at a timescale of a few days, as seen in Epoch 7. Examining with various backgrounds sampled from different source-free regions, we found that this result is robust. With the column absorption fixed at $1.1 \times 10^{20} \mathrm{~cm}^{-2}$, its spectrum can be described by a power-law model with a photon index of $\Gamma=2.19_{-0.43}^{+0.47}$. Its absorption-corrected flux is at the level of $f_{x} \sim 6.8 \times 10^{-15} \mathrm{erg} \mathrm{cm}^{-2} \mathrm{~s}^{-1}(0.3-8 \mathrm{keV})$.

In Figure 7, we also noted that a counter-protrusion apparently extends toward the northwestern direction in both epochs. Its brightness is about a factor of two lower than that of the southeastern protrusion. However, such a feature is only significant at an $\sim 2 \sigma$ level, and therefore we cannot confirm its existence.

We also investigated the spatial nature of the X-ray emission at the pulsar position through a $2 \mathrm{D}$ image fitting with the aid of Sherpa. We aimed to probe if there is any indication of the compact nebula associated with Geminga. For each epoch, we fit the subpixel-resolution image with a composite model of a 2D Gaussian model and a horizontal plane to account for the compact source and the background, respectively. Except for Epoch 2, the spatial distributions of the X-ray photons from Geminga in all the other observations conform with that expected from a point source. In Figure 9, the close-up unsmoothed image of Epoch 2 with a field of $15^{\prime \prime} \times 15^{\prime \prime}$ centered at the pulsar is shown. The distribution of the photons is apparently elongated. The 2D fitting yields a FWHM of 1 ." $35 \pm 0$ ". 02 with respect to the best-fit major axis, which is 

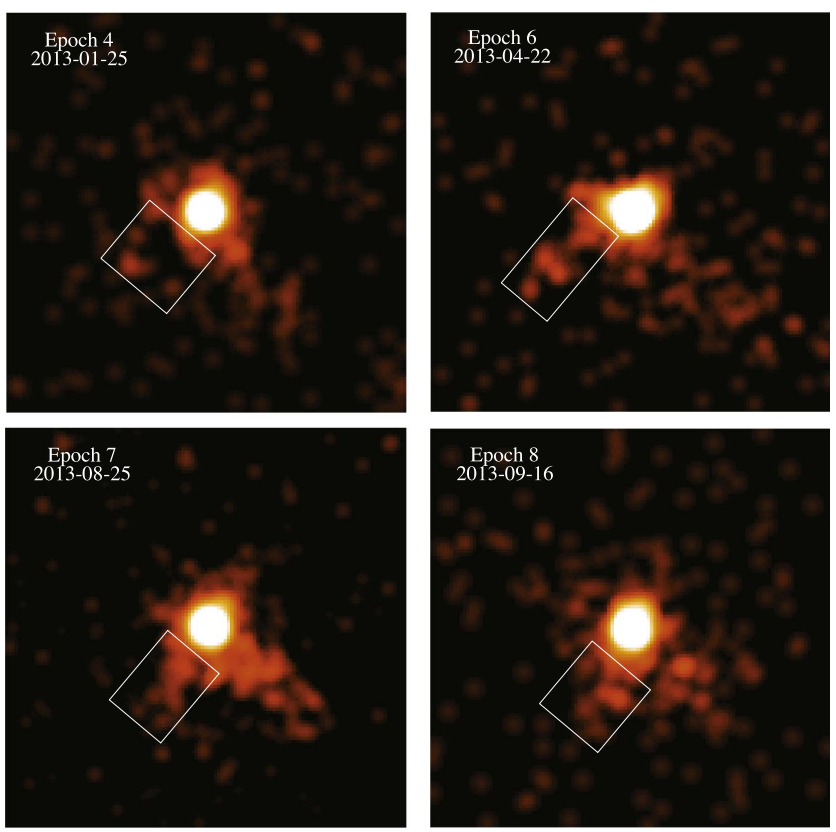

Figure 6. Candidates for compact extended features around Geminga as identified by visual inspection. The possible protrusions are highlighted by the boxes in each image.

along the proper-motion direction. We subsequently examined the spectrum of the pulsar from Epoch 2 to see if there is any evidence of an additional contribution from the compact nebula. Adopting the same spectral models used in Mori et al. (2014), we found that all the parameters inferred from fitting the Epoch 2 spectrum are consistent with them, and there is no indication that an extra spectral component is required.

We also reexamined the arc-like feature in Epochs 1 and 2 as reported by Pavlov et al. (2010), adopting a source region similar to that in their study (cf. Figure 2 in their paper). We found that the $\mathrm{S} / \mathrm{Ns}$ of this feature are only $\sim 3.4 \sigma-4 \sigma$. These are consistent with the results of Pavlov et al. (2010). However, it is below the $5 \sigma$ criterion we adopted to firmly discriminate genuine features from the background fluctuation in this work. Also, we did not find any solid evidence for such a feature in the other observations. Therefore, we will not further consider this feature in our work.

\subsection{Outer Tails}

For investigating the variability of both outer tails, Epoch 1 data are excluded in this analysis, as they do not cover the whole feature. In Figure 10, we show the time sequence of $5^{\prime} \times 5^{\prime}$ exposure-corrected images from Epoch 2 to Epoch 8 . We computed the X-ray contours from the Epoch 3 image, which has a high $\mathrm{S} / \mathrm{N}(\sim 9.4 \sigma)$, and the whole feature is not intervened by any CCD gap. We adopted these contours as a reference and overlaid them on all images to investigate if there are any morphological variations at different epochs.

In Epoch 4, both the northern and southern tails are found to be apparently different from those in the other epochs. The southern tail appears to be shorter in Epoch 4. However, this is due to the fact that a large fraction of this feature fell in the CCD gap. By contrast, the northern tail appears to be longer in this epoch. But the $\mathrm{S} / \mathrm{N}$ of this feature in Epoch 4 is only $\sim 2.3 \sigma$; hence, its variability is inconclusive. In view of this, we conclude that the morphologies of both outer tails from Epochs 2 to 6 are found to be consistent.

In Epochs 7 and 8, the southern tail is found to be significantly twisted with respect to the contours (see Figure 10). An S-shaped structure can be clearly seen in these images. Such morphological change has been displayed in different images obtained in two consecutive epochs, indicating that it is not a result of background fluctuations. For highlighting the twisted feature, we combined the exposurecorrected images of Epochs 7 and 8 in Figure 11 so as to enhance its significance. This morphological variation is different from that of the axial tail; there is no propagation that can be found in the southern outer tail, as the far end of this feature appears to fix at the same position in Epochs $7+8$ and 3 . Instead, obvious lateral displacement can be seen by comparing these images (use the blink feature in interactive Figure 11 to illustrate this displacement). Comparing the images in Epochs 3 and 7 (separated by $\sim 9$ months), the maximum deviation of the southern tail is $\sim 0.5$ (illustrated in Figure 11). At a distance of $250 \mathrm{pc}$, this lateral displacement corresponds to a physical scale of $\sim 10^{17} \mathrm{~cm}$. These imply that the twisting of the southern tail occurred at a speed of $\sim 0.2 c$.

To investigate if there is any short term variability on the order of a few days as in the cases of the axial tail and circumstellar diffuse emission, we have also searched for the possible morphological variability of the outer tails among the individual observations in Epochs 7 and 8. Nevertheless, we do not find any significant short-term variability.

For the spectral analysis, we first cross-checked the epochaveraged outer tails obtained by Posselt et al. (2017) with the simultaneous fit of all the data from Epochs 2 to 8 . For each epoch, we adopted a region so as to fit the morphology of each tail and avoid the CCD gaps. Here $\Gamma=0.94 \pm 0.20$ (northern tail) and $\Gamma=1.02 \pm 0.09$ (southern tail) are derived from our independent analysis and consistent with the values obtained by Posselt et al. (2017; cf. Table 3 in their paper). For the southern tail, while the flux is comparable to that obtained by Pavlov et al. (2010) based on Epoch 2 data, the epoch-averaged photon index obtained by Posselt et al. (2017) and us is apparently harder than that reported by Pavlov et al. (2010), namely $\Gamma=1.59_{-0.28}^{+0.30}$. This indicates the possible spectral hardening in the later epochs. However, the search for the spectral variation of the outer tails has not been considered in Posselt et al. (2017).

We proceeded to examine the spectral properties of the southern tail in each epoch. The results are summarized in Table 3. The corresponding background-subtracted spectra and fitting residuals are shown in Figure 12. The spectral parameters obtained from Epoch 2 are consistent with those reported by Pavlov et al. (2010). In Epochs 4-7, the best-fit photon indices appear to become smaller. This explains the discrepancy between Posselt et al. (2017) and Pavlov et al. (2010). Particularly in Epoch 7, the spectrum became the hardest among all epochs when the tail was significantly twisted (see Figure 11). Comparing with the epoch-averaged result, the photon index in this epoch is smaller by $\sim 3 \sigma$. In Epoch 8, the spectrum becomes softer again.

For the northern tail, the $\mathrm{S} / \mathrm{N}$ is $<4 \sigma$ in images of all seven epochs. Hence, we are not able to conclusively quantify if there is any morphological variability. 

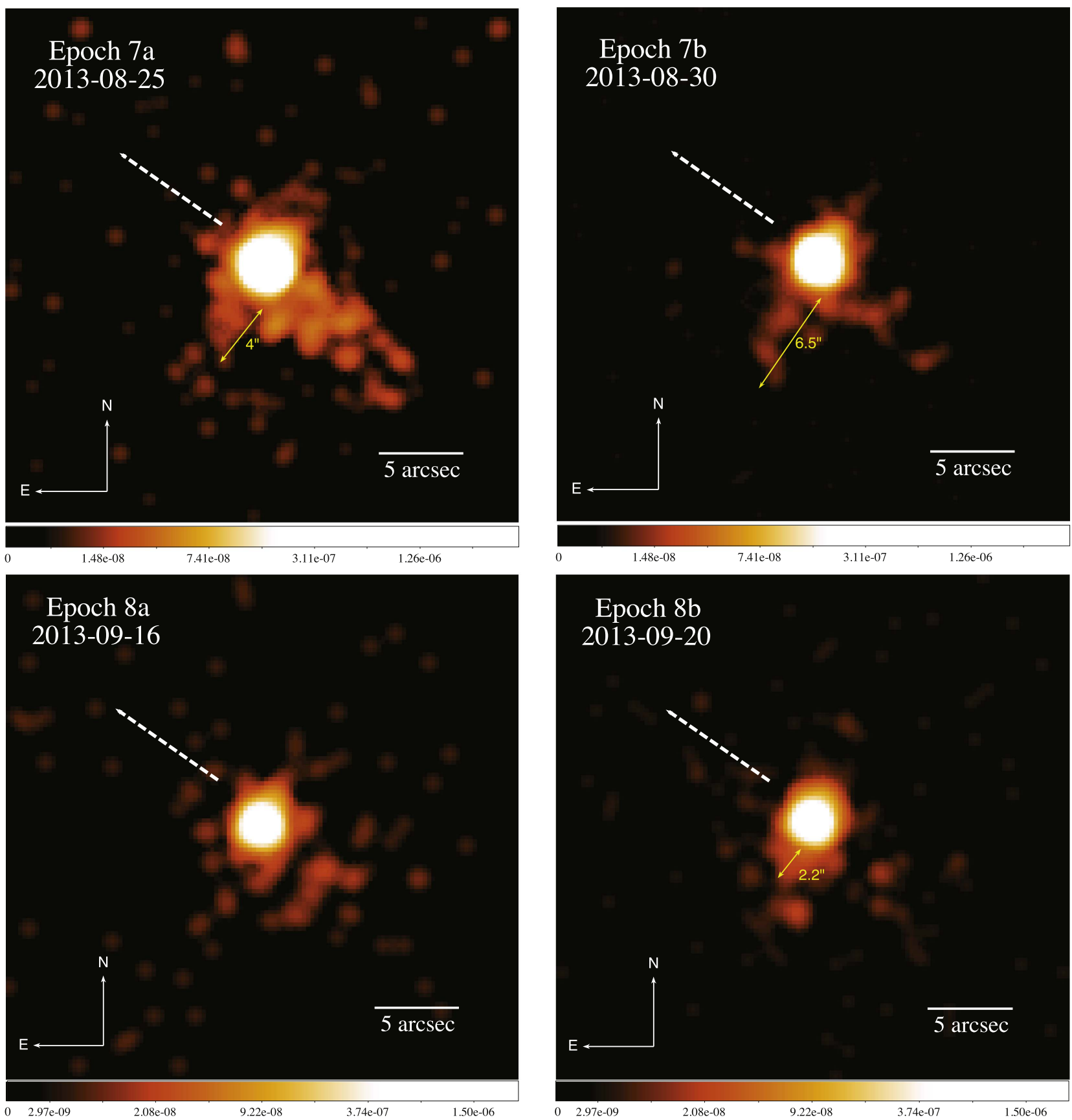

Figure 7. Exposure-corrected subpixel-resolution images $\left(0 . ! 25 \times 0 . .25\right.$ for 1 pixel) in $0.5-7 \mathrm{keV}$ of $30^{\prime \prime} \times 30^{\prime \prime}$ centered on Geminga as observed by Chandra in 2013 August and September. The images are smoothed by a Gaussian kernel of $1^{\prime \prime}$. The images of subframes in each epoch are adjusted to the same logarithmic color scale for comparison. The morphological variation of the axial tail and the circumstellar diffuse emission can be clearly seen. The angular sizes of the southeastern protrusion are illustrated in each image by the yellow double arrow. The scale bar at the bottom of each image shows the pixel values in units of photons $\mathrm{cm}^{-2} \mathrm{~s}^{-1}$. The interactive figure will blink between all four epochs.

We further investigated the spectral hardening of the southern tail. From Figure 1, it appears that its X-ray emission is harder in the region further away from the pulsar than that close to the pulsar. This motivates us to performed a spatially resolved analysis to investigate if there is any spatial variation of spectral properties and pinpoint the origin of the spectral hardening. We divided each tail into two halves, with one closer to the pulsar ("front") and the other further away from it ("rear"). The results are summarized in Table 4. The corresponding background-subtracted spectra and fitting residuals are shown in Figure 13.

There is an indication of spectral softening along the southern tail found in Epochs 2 and 3. On the contrary, spectral hardening toward the end of the feature has been observed from Epoch 4 to Epoch 8. Particularly in Epoch 7, the difference of $\Gamma$ inferred in the front and rear spectra is $\sim 3 \sigma$.

We further examined the spectral hardening along the southern tail from Epoch 4 to Epoch 8. By simultaneously fitting their front 

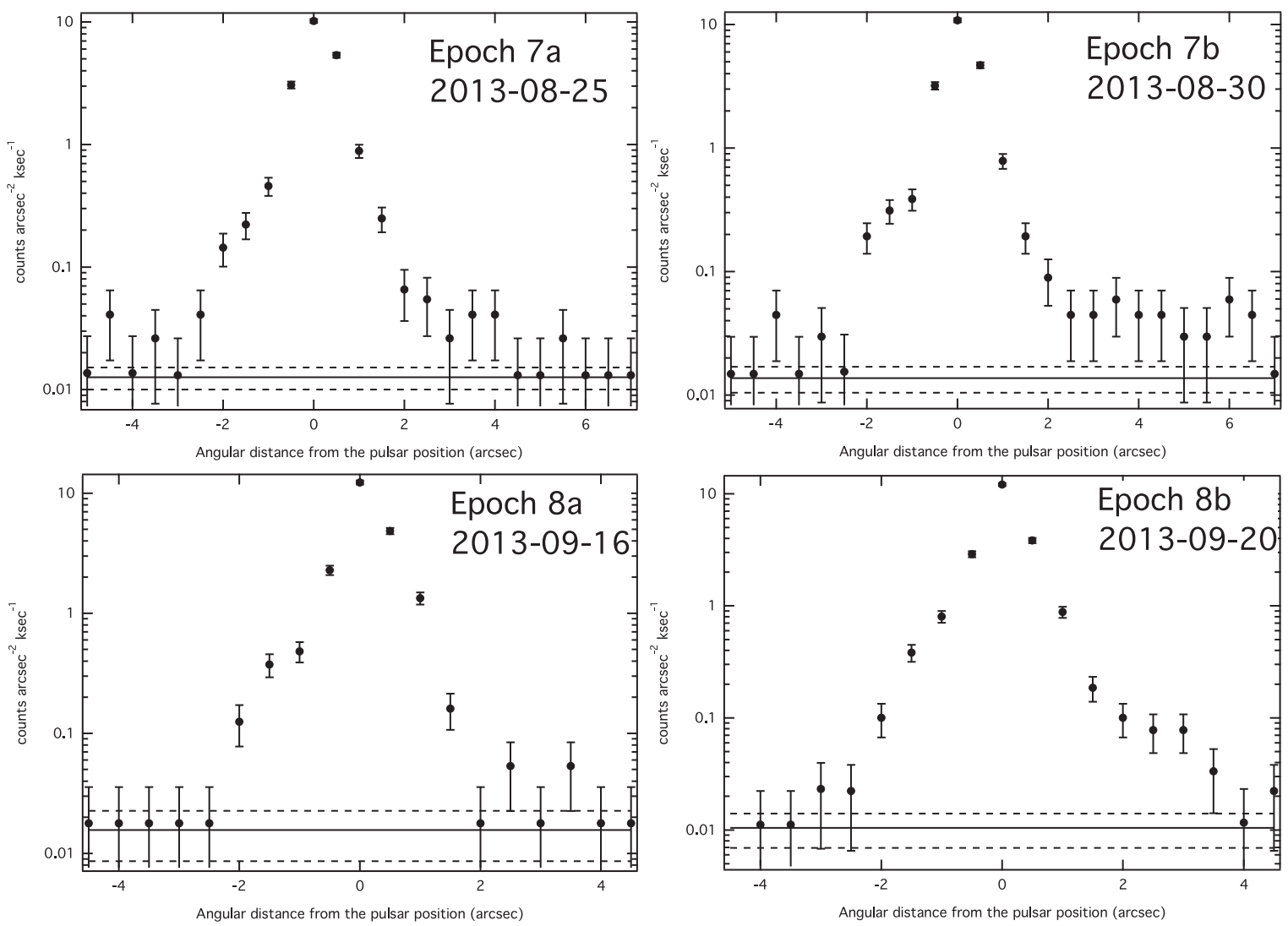

Figure 8. Brightness profiles along the southeastern protrusion (cf. Figure 7) as computed from the unsmoothed subpixel-resolution images in Epochs 7a, 7b, 8a, and $8 \mathrm{~b}$. The positive and negative direction of the $x$ axis correspond to the orientations of southeast and northwest from the pulsar position. The horizontal solid and dashed lines represent the background and its $1 \sigma$ uncertainty at each epoch. The southeastern protrusion appears as the excess above the background level inthe positive direction of the $x$ axis.

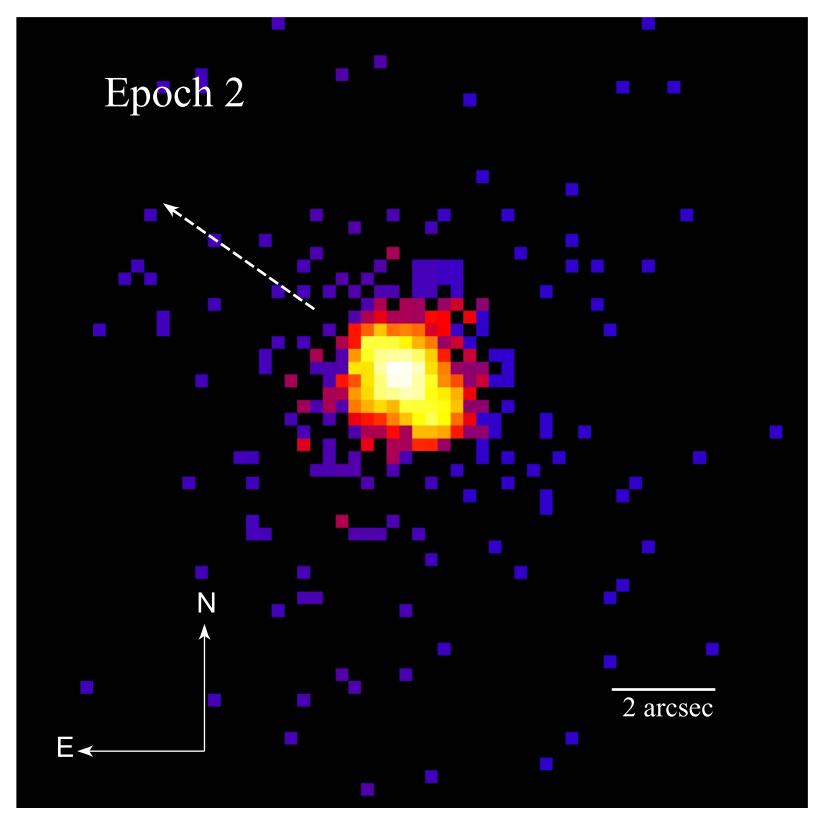

Figure 9. Exposure-corrected subpixel-resolution raw image (image bin size $=0.25)$ in the energy range of $0.5-7 \mathrm{keV}$ of a $15^{\prime \prime} \times 15^{\prime \prime}$ field centered on Geminga as observed by Chandra ACIS-I on 2007 August 27 (i.e., Epoch 2). The distribution of the X-ray photons from the pulsar is elongated along the orientation of its proper motion.

spectra in these five epochs, we obtained a photon index of $\Gamma=1.32_{-0.16}^{+0.17}$. Similarly, this yields a photon index of $\Gamma=$ $0.84 \pm 0.14$ for the rear part. The corresponding confidence contours are shown in Figure 14, which clearly shows the significant spectral variation along the southern outer tail.

We have cross-checked all the aforementioned results of spectral hardening with backgrounds sampled from different low-count regions. The results of all the independent spectral fits are found to be consistent within the tolerance of their statistical uncertainties.

We note that Posselt et al. (2017) did not find any spectral variation along the southern tail. This can be ascribed to the fact that they only analyzed the combined spectra. As there is evidence of spectral softening in Epochs 2-3, the spectral variation can be averaged out in the combined spectra.

We have also examined the front and rear spectra of the northern tail with all the observations combined, and we have noticed that the best-fit photon index in the rear part, $\Gamma \sim 1.3$, is larger than that of the front part, $\Gamma \sim 0.6$. This might indicate a spectral softening along the feature. However, in view of the larger statistical uncertainties, we cannot unambiguously conclude this with the existing data.

\section{Summary and Discussion}

In this investigation, we have searched for the X-ray variabilities of the PWN powered by Geminga. Apart from cross-checking the epoch-averaged results recently reported by Posselt et al. (2017), we report a number of new findings in our variability analysis, which are summarized as follows. 

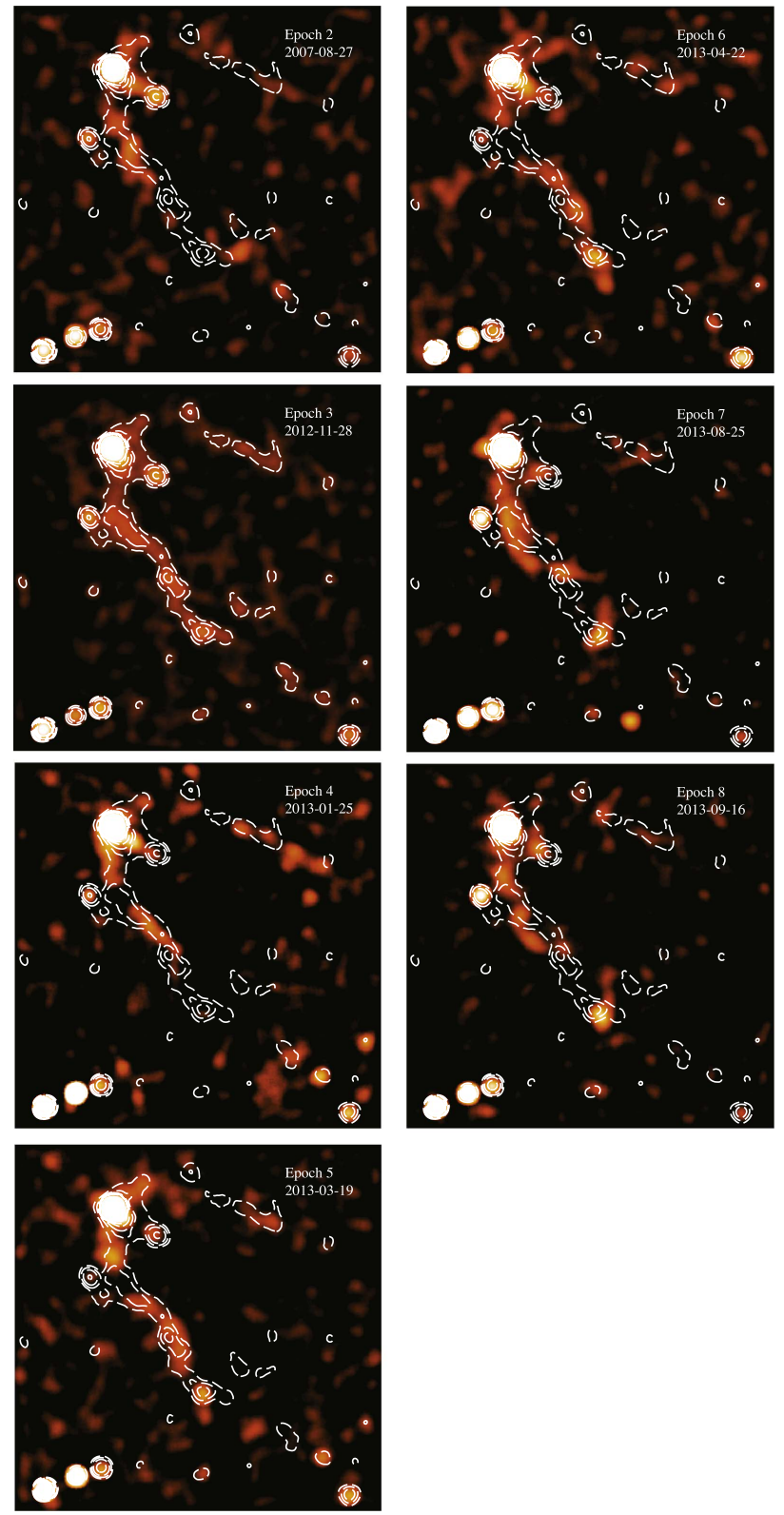

Figure 10. Time sequence of Chandra $5^{\prime} \times 5^{\prime}$ exposure-corrected images $(0.5-7 \mathrm{keV})$ that cover the outer tails of Geminga. The observation in 2004 is excluded in this analysis, as it did not cover the whole feature. All images are smoothed with a Gaussian kernel of $\sigma=6^{\prime \prime}$. The contours computed from the images obtained in Epoch 3 are overlaid on all images for comparing the morphology of outer tails at different epochs.

1. Discovery of spectral and morphological variations of the axial tail in 2013 August/September, which took place on a timescale as short as a few days (Figures 5 and 7).

2. Confirming the existence of the variable southeastern protrusion that connects the pulsar and the southern outer tail (Figures 7 and 8).

3. Finding the spatial distribution of the photons from the pulsar to be elongated in 2007 August (Figure 9).

4. Confirming the wiggling of the southern outer tail that took place on a timescale of a few months (Figure 11).

5. Discovery of the spectral hardening of the southern outer tail from 2013 January to August (Table 3) and identifying the spectral hardening that occurred at the rear end of the feature (Figures 1 and 14).

All these results can be interpreted in the context of a general model of PWN structure that consists of a torus around the pulsar, jets along the spin axis, and a synchrotron nebula resulting from the postshock flow (cf. Gaensler \& Slane 2006 for a detailed review). In the following discussion, we assume that the compact elongated structure (Figure 9), southeastern protrusion+outer tails (Figures 7 and 11), and axial tail (Figures 2 and 7) correspond to the torus, jets, and bow-shock nebula, respectively.

Among all these, the most spectacular results are the morphological and spectral variability of the southern outer tail. Both outer tails can be interpreted as bipolar outflows from the pulsar that are bent by the ISM ram pressure. Such significant jet bending has not been seen in other PWNe. Our interpretation is supported by the discovery of the collimated southeastern protrusion that is connected to the southern outer tail (Figure 7). The protrusion is likely to be a collimated jet powered by the pulsar.

In this scenario, the significant bending of the jets indicates that the ram pressure of the ISM is comparable with that of the jet. This structure is similar to the bent jets observed in some radio galaxies (e.g., McBride \& McCourt 2014).

The ram pressure of the jet $P_{j}$ can be expressed as

$$
P_{j} \sim \frac{\dot{E}}{\pi \theta_{j}^{2} r_{j}^{2} c}
$$

where $\theta_{j}$ and $r_{j}$ are the open angle and the length of the jet, respectively. In contrast, the ram pressure of the ISM $P_{a}$ is given by

$$
P_{a} \sim \rho_{\mathrm{ISM}} v_{\mathrm{psr}}^{2}
$$

where $\rho_{\text {ISM }}$ and $v_{\mathrm{psr}}$ are the ISM density and the pulsar's velocity, respectively. For $\rho_{\text {ISM }} \sim 2 \times 10^{-24} \mathrm{~g} \mathrm{~cm}^{-3}$ and $v_{\text {psr }} \sim$ $200 \mathrm{~km} \mathrm{~s}^{-1}$, the ISM ram pressure is on the order of $P_{a} \sim$ $10^{-9} \mathrm{erg} \mathrm{cm}^{-3}$. Assuming $P_{j} \sim P_{a}$ and $r_{j} \sim 0.1 \mathrm{pc}$, it implies the open angle of the jet with a few degrees.

From the theory of the jet-bending model of galaxies (Begelman et al. 1979), the curvature radius $R_{c}$ of the bent jet can be expressed as

$$
R_{c} \sim \frac{P_{j}}{P_{a}} h,
$$

where $h$ is the scale height of the jet perpendicular to the motion of the jet. For $P_{j} \sim P_{a}, R_{c} / h \sim 1$ implies a bending angle of $\sim 60^{\circ}$, which is not far from the observed deviation (see Figure 11).

In Epochs 7a, 7b, and 8b, we also noted that a counterprotrusion apparently extends toward the northwestern direction. Its brightness is about a factor of two lower than that of the southeastern protrusion. The difference in brightness between this putative counterjet and the southeastern protrusion can be explained by Doppler boosting. We can place a constraint on the angle $\zeta$ between the spin axis and the line of 


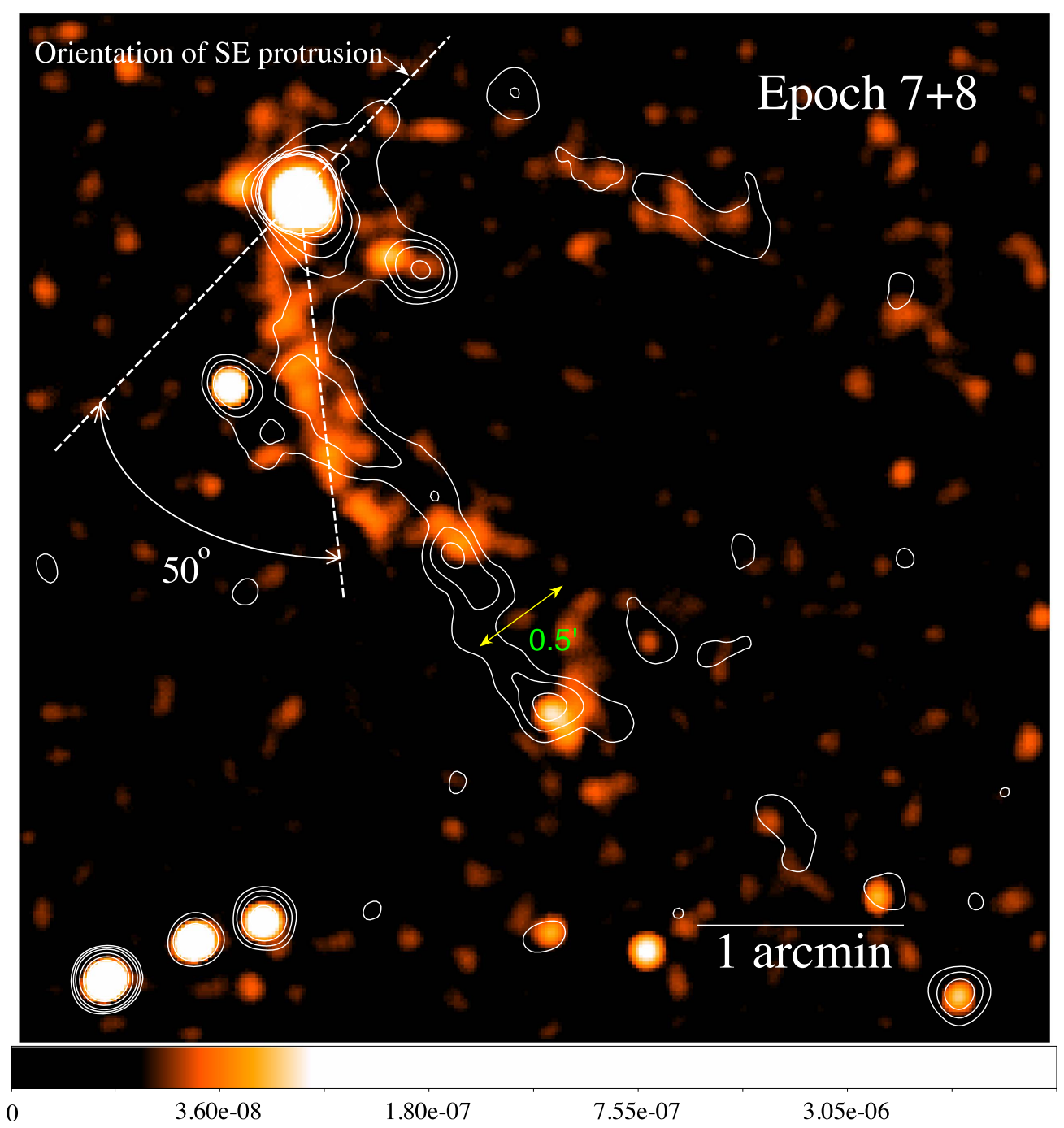

Figure 11. The $5^{\prime} \times 5^{\prime}$ exposure-corrected image (0.5-7 keV; $1^{\prime \prime} \times 1^{\prime \prime}$ for 1 pixel) that covers the outer tails of Geminga as observed by Chandra in 2013 August and September (Epochs 7+8). It has been smoothed with a Gaussian kernel of $\sigma=6^{\prime \prime}$. The contours computed from the images obtained in 2012 November/December (Epoch 3) are overlaid for visualizing the morphological variation of the outer tails. The wiggling motion of the southern outer tail can be found through this comparison. The maximum lateral displacement of $\sim 0^{\prime} 5$ at the rear part of the tail is shown by the yellow double arrow. The deviation of the southern outer tail with respect to the southeastern protrusion is also illustrated. The scale bar at the bottom shows the pixel values in units of photons $\mathrm{cm}^{-2} \mathrm{~s}^{-1}$. The interactive figure will blink between the frames in Epochs 3 and $7+8$.

Table 3

Spectral Properties of the Southern Tail in Different Epochs

\begin{tabular}{|c|c|c|c|c|c|c|c|c|}
\hline & $\begin{array}{c}A_{S} \\
\left(\operatorname{arcsec}^{2}\right)\end{array}$ & $\begin{array}{c}N_{\text {net }} \\
\text { counts }\end{array}$ & $\begin{array}{c}N_{\text {bkg }} \\
\text { counts }\end{array}$ & $\alpha$ & $\begin{array}{l}\mathrm{S} / \mathrm{N} \\
(\sigma)\end{array}$ & $\Gamma$ & $\begin{array}{c}F_{\text {obs }} \\
\left(10^{-14} \mathrm{erg} \mathrm{cm}^{-2} \mathrm{~s}^{-1}\right)\end{array}$ & $\begin{array}{c}L_{\text {unabsorb }} \\
\left(10^{29} \mathrm{erg} \mathrm{s}^{-1}\right)\end{array}$ \\
\hline Epoch 2 & 5881 & 198 & 1329 & 0.49 & 5.8 & $1.66_{-0.32}^{+0.37}$ & $3.12_{-0.61}^{+0.70}$ & $2.34_{-0.45}^{+0.53}$ \\
\hline Epoch 3 & 5001 & 311 & 3172 & 0.20 & 9.4 & $1.43_{-0.24}^{+0.27}$ & $3.31_{-0.50}^{+1.25}$ & $2.48_{-0.37}^{+0.93}$ \\
\hline Epoch 4 & 1816 & 126 & 3264 & 0.08 & 6.2 & $1.08_{-0.29}^{+0.31}$ & $1.86_{-0.36}^{+0.39}$ & $1.39_{-0.27}^{+0.29}$ \\
\hline Epoch 5 & 3726 & 224 & 3079 & 0.14 & 8.3 & $0.87_{-0.23}^{+0.24}$ & $4.23_{-0.59}^{+0.62}$ & $3.16_{-0.44}^{+0.46}$ \\
\hline Epoch 6 & 2486 & 279 & 4263 & 0.08 & 11.2 & $0.81 \pm 0.17$ & $4.17_{-0.50}^{+0.52}$ & $3.12_{-0.38}^{+0.39}$ \\
\hline Epoch 7 & 4730 & 333 & 3887 & 0.14 & 10.7 & $0.41 \pm 0.20$ & $6.18_{-0.76}^{+0.79}$ & $4.62_{-0.57}^{+0.59}$ \\
\hline Epoch 8 & 4485 & 291 & 5013 & 0.10 & 9.9 & $1.25_{-0.20}^{+0.21}$ & $3.90_{-0.49}^{+0.51}$ & $2.92_{-0.36}^{+0.38}$ \\
\hline
\end{tabular}

sight. The brightness ratio $f_{b}$ can be written as

$$
f_{b}=\left(\frac{1+\beta \cos \zeta}{1-\beta \cos \zeta}\right)^{\Gamma+2},
$$

where $\Gamma$ is the photon index of the jet and $\beta$ is the outflow velocity in units of $c$.

From comparing the images in Epochs $7 \mathrm{a} / 7 \mathrm{~b}$ and $8 \mathrm{a} / 8 \mathrm{~b}, \beta$ is found to be $\sim 0.7-0.8$. In view of the low significance of the 

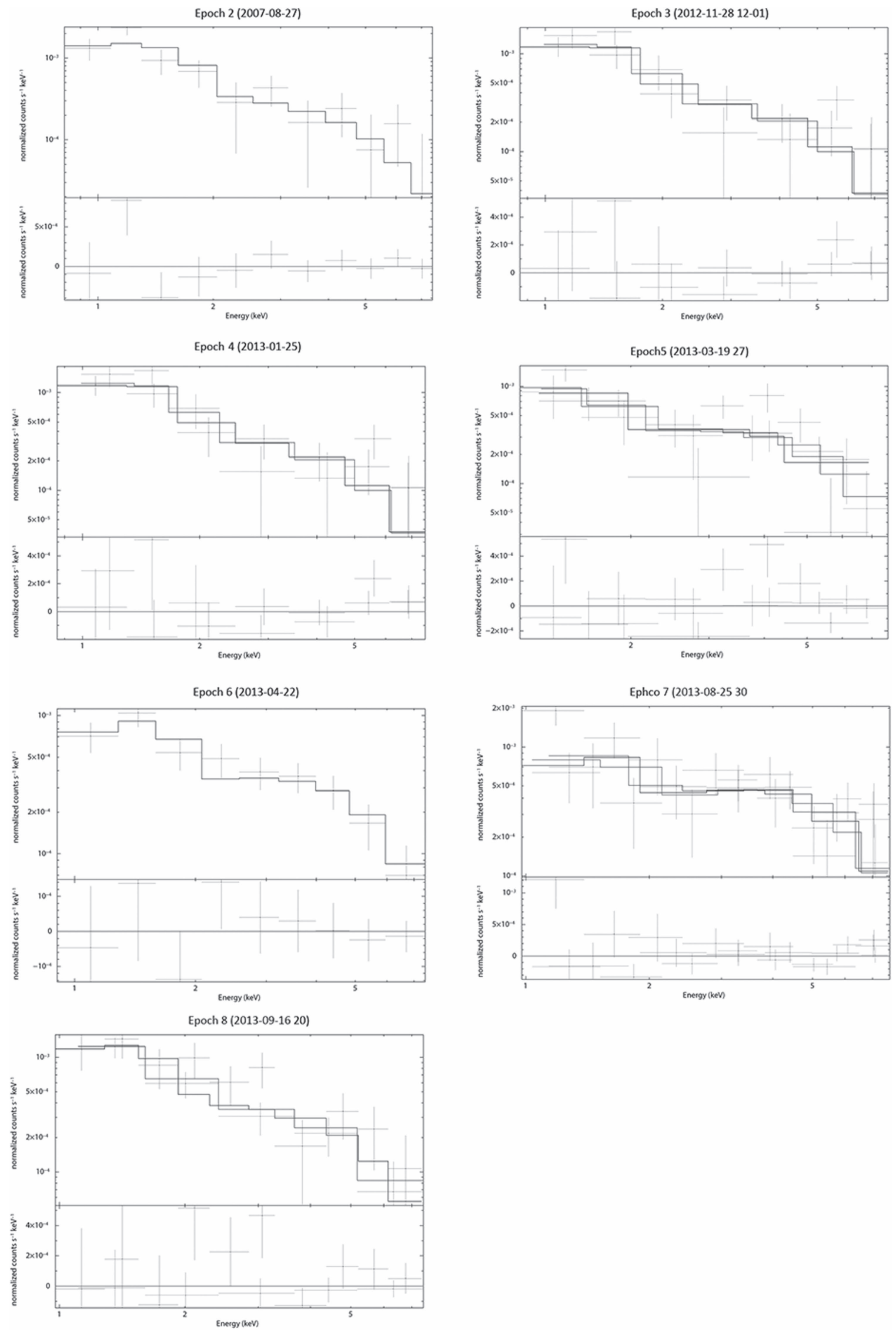

Figure 12. Background-subtracted energy spectra of the southern outer tail (upper panels) with the best-fit power-law models (solid lines) in different epochs. The best-fit spectral parameters are given in Table 3. The fitting residuals are also shown (lower panels). 
Table 4

Results from the Spatially Resolved Spectral Analysis of the Outer Tails

\begin{tabular}{|c|c|c|c|c|c|c|c|c|}
\hline & $\begin{array}{c}A_{S} \\
\left(\operatorname{arcsec}^{2}\right)\end{array}$ & $\begin{array}{c}N_{\text {net }} \\
\text { counts }\end{array}$ & $\begin{array}{l}N_{\text {bkg }} \\
\text { counts }\end{array}$ & $\alpha$ & $\begin{array}{l}\mathrm{S} / \mathrm{N} \\
(\sigma)\end{array}$ & $\Gamma$ & $\left(10^{-14} \mathrm{erg} \mathrm{cm}^{F_{\text {obs }}} \mathrm{s}^{-1}\right)$ & $\begin{array}{c}L_{\text {ynabsorb }} \\
\left(10^{29} \mathrm{erg} \mathrm{s}^{-1}\right)\end{array}$ \\
\hline \multicolumn{9}{|c|}{ Northern Tail } \\
\hline Merged (Front) & 1844 & 333 & 8972 & 0.18 & 7.1 & $0.61 \pm 0.27$ & $0.93_{-0.15}^{+0.16}$ & $0.69_{-0.11}^{+0.12}$ \\
\hline Merged (Rear) & 3236 & 231 & 8972 & 0.31 & 3.7 & $1.30_{-0.30}^{+0.34}$ & $0.68_{-0.15}^{+0.16}$ & $0.51_{-0.11}^{+0.12}$ \\
\hline \multicolumn{9}{|c|}{ Southern Tail } \\
\hline Epoch 2 (Front) & 1974 & 123 & 1329 & 0.17 & 6.3 & $1.36_{-0.34}^{+0.40}$ & $2.10_{-0.46}^{+0.54}$ & $1.57_{-0.34}^{+0.40}$ \\
\hline Epoch 2 (Rear) & 3282 & 64 & 1329 & 0.27 & 2.7 & $2.12_{-0.62}^{+0.89}$ & $1.01_{-0.27}^{+0.38}$ & $0.76_{-0.20}^{+0.28}$ \\
\hline Epoch 3 (Front) & 2951 & 239 & 3172 & 0.12 & 9.2 & $1.33_{-0.25}^{+0.28}$ & $2.34_{-0.38}^{+0.41}$ & $1.75_{-0.28}^{+0.30}$ \\
\hline Epoch 3 (Rear) & 2149 & 102 & 2535 & 0.10 & 5.2 & $1.87_{-0.81}^{+1.37}$ & $0.71_{-0.22}^{+0.31}$ & $0.53_{-0.16}^{+0.23}$ \\
\hline Epoch 4 (Front) & 591 & 68 & 3264 & 0.03 & 5.4 & $1.73_{-0.44}^{+0.52}$ & $0.60_{-0.14}^{+0.17}$ & $0.45_{-0.11}^{+0.12}$ \\
\hline Epoch 4 (Rear) & 1225 & 58 & 3264 & 0.05 & 3.7 & $1.07_{-0.39}^{+0.45}$ & $1.03_{-0.29}^{+0.33}$ & $0.77_{-0.22}^{+0.24}$ \\
\hline Epoch 5 (Front) & 658 & 71 & 3079 & 0.03 & 5.8 & $1.40_{-0.46}^{+0.51}$ & $1.08_{-0.21}^{+0.24}$ & $0.81_{-0.16}^{+0.18}$ \\
\hline Epoch 5 (Rear) & 3068 & 153 & 3079 & 0.12 & 6.5 & $0.66 \pm 0.27$ & $3.00_{-0.54}^{+0.57}$ & $0.24_{-0.40}^{+0.43}$ \\
\hline Epoch 6 (Front) & 466 & 74 & 4263 & 0.01 & 6.4 & $1.28_{-0.36}^{+0.44}$ & $0.83_{-0.19}^{+0.21}$ & $0.62_{-0.14}^{+0.16}$ \\
\hline Epoch 6 (Rear) & 2020 & 205 & 4263 & 0.06 & 9.3 & $0.70 \pm 0.20$ & $3.08_{-0.44}^{+0.46}$ & $2.31_{-0.33}^{+0.35}$ \\
\hline Epoch 7 (Front) & 2476 & 203 & 3887 & 0.08 & 8.9 & $0.70_{-0.21}^{+0.20}$ & $3.29_{-0.47}^{+0.48}$ & $2.46_{-0.35}^{+0.36}$ \\
\hline Epoch 7 (Rear) & 2678 & 129 & 3887 & 0.08 & 6.0 & $-0.13_{-0.59}^{+0.50}$ & $2.66_{-0.62}^{+0.68}$ & $1.99_{-0.475}^{+0.51}$ \\
\hline Epoch 8 (Front) & 2298 & 173 & 5013 & 0.05 & 8.1 & $1.57_{-0.29}^{+0.32}$ & $1.84_{-0.29}^{+0.32}$ & $1.37_{-0.22}^{+0.24}$ \\
\hline Epoch 8 (Rear) & 2189 & 118 & 5013 & 0.05 & 6.0 & $1.01_{-0.29}^{+0.31}$ & $1.67_{-0.34}^{+0.37}$ & $1.25_{-0.26}^{+0.27}$ \\
\hline
\end{tabular}

counterjet $(\sim 2 \sigma)$, we consider a lower limit of $f_{b}$ to be $>2$. The photon index of the jet has a large uncertainty, and it might vary at different epochs. It spans a range of $\Gamma \sim 0.7-2.7$ in Epochs $7 \mathrm{~b}$ and $8 \mathrm{~b}$. With these observational inputs, we place an upper limit of $\zeta$ at $<80^{\circ}$. This is close to the value inferred by modeling the $\gamma$-ray light curve of Geminga (Zhang \& Cheng 2001).

The orientation of the jet and counterjet is almost perpendicular to the proper-motion direction. Assuming the spin axis of Geminga is aligned with the jet/counterjet, its spin vector and velocity vector are apparently orthogonal to each other. This is different from the spin-velocity alignment seen in many other pulsars (Noutsos et al. 2012). The orthogonal spinvelocity configuration of Geminga might be a result of a single localized momentum impulse imparted on the neutron star when it was born (Spruit \& Phinney 1998).

Interpreting this protrusion as a jet suggests the possible presence of a torus around the pulsar. A torus-plus-jet structure has been seen in many other pulsars, such as Crab (Weisskopf et al. 2000; Gaensler \& Slane 2006). In searching for such a compact structure, we have identified an elliptical feature around the pulsar in Epoch 2 (Figure 9).

This feature can be interpreted as a tilted torus around Geminga. This scenario is supported by the presence of the jetlike protrusion along the toroidal axis. Relativistic MHD (RMHD) simulations have shown that the energy of the pulsar wind is concentrated toward the equator. When the wind interacts with the circumstellar medium, a termination shock can emerge. In the downstream of this shock, plasma flows into the equatorial region and thus forms a torus (cf. Figure 4 in Porth et al. 2017). The plasma also flows to the polar region. In the presence of the magnetic hoop stress, the jets perpendicular to the torus can be formed (for a recent review of the RMHD study of PWNe, see Porth et al. 2017).
Under this assumption, the termination shock radius $R_{s}$ can be estimated by the balance between the ram pressure of the pulsar wind and the magnetic pressure $B^{2} / 8 \pi$ of the PWN:

$$
R_{s} \simeq\left(\frac{\dot{E}}{B^{2} c}\right)^{1 / 2}
$$

At a distance of $250 \mathrm{pc}$, the width of $3 \sigma=1.15$ inferred from image fitting corresponds to a physical size of $5.6 \times 10^{15} \mathrm{~cm}$, which is about two orders smaller than the X-ray ring of the Crab Nebula. Taking this as the estimate of $R_{s}$ implies a magnetic field of $B \sim 195 \mu \mathrm{G}$, which is typical for PWNe (Gaensler \& Slane 2006). As this elliptical feature can only be seen in one observation, it is very likely that the feature is also variable. The torus of Crab has been found to shrink (Mori et al. 2004). In case the elliptical feature of Geminga detected in 2007 August shrinks by at least a factor of two, it will not be able to be resolved by any state-of-the-art X-ray telescope.

Variability timescales ranging from a few days to a few months have been found in various spatial components of Geminga's PWN. These timescales should shed light and put constraints on modelings of this complex system and the MHD processes there.

The southeastern jetlike protrusion, for instance, is clearly variable. As it can only be detected in 2013 August and September, this indicates that the outflow was enhanced in these two epochs. This might be related to the observed wiggling motion of the outer southern tail in these epochs. A similar wiggling motion has also been observed in the jets of Vela and Crab (Pavlov et al. 2003; Mori et al. 2004). These variations are found on the order of a few tens of percent of the speed of light. 

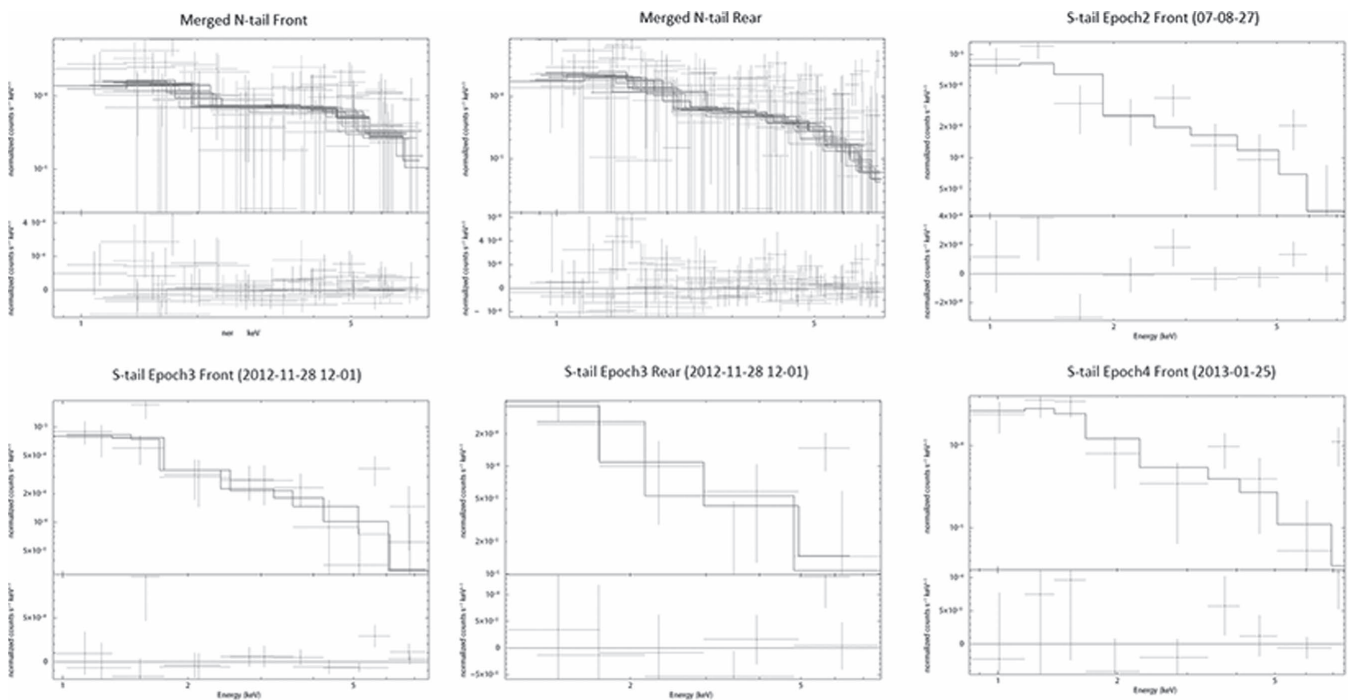

S.tail Epochs Rear (2013-03-19 27)
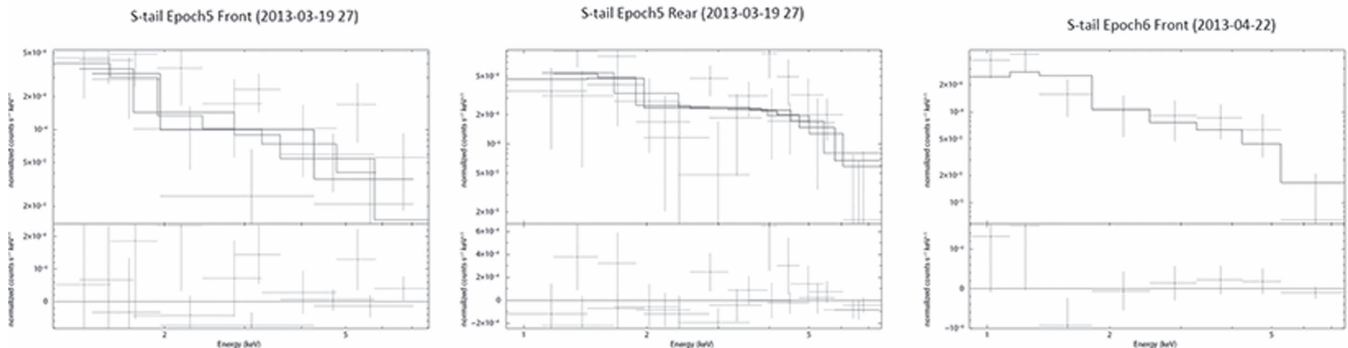

S.tail Epoch7 Rear (2013-08.25 30)
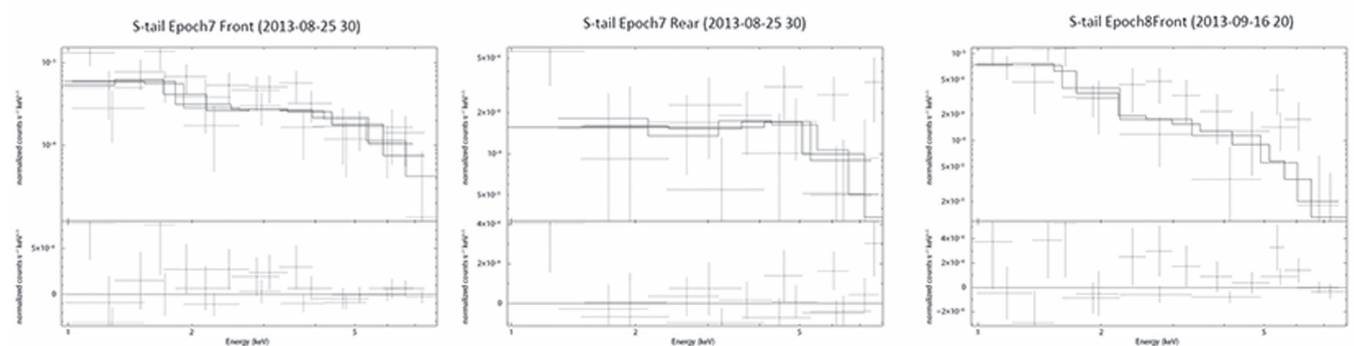
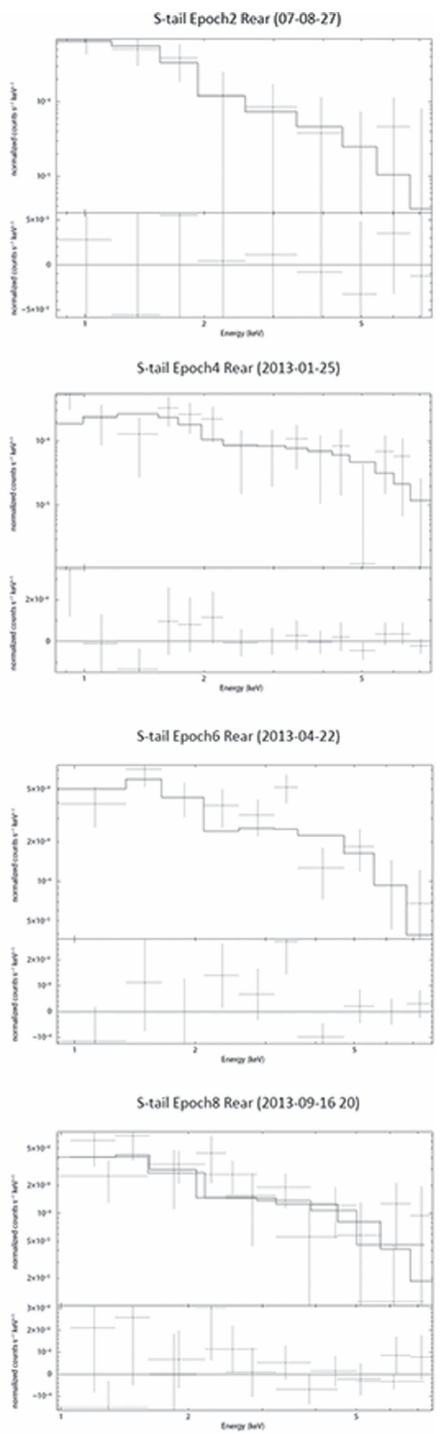

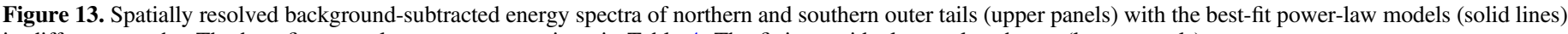
in different epochs. The best-fit spectral parameters are given in Table 4. The fitting residuals are also shown (lower panels).

The nature and origin of the variabilities of the outer and axial tails were conjectured before. Pavlov et al. (2010) and Posselt et al. (2017), for instance, examined both the shell model of shocked wind and the jetlike outflow model; in the shell model, the variabilities could result from shear instabilities, while in the outflow model, they could be induced by internal instabilities or external environmental effects (i.e., sweepback by external ram pressure).

For the southern outer tail, different from the axial tail, it appears that the twisted structure does not have any propagation (see Figure 11). This indicates that the natures of the variabilities of these two PWN components are different. The absence of propagation in the case of the southern outer tail suggests that it can possibly be a helical structure resulting from torsional Alfv́en waves. Similar phenomena have been seen in the solar coronal jets (Srivastava et al. 2017; Szente et al. 2017), though the scale of the Geminga jet is much larger.
While a rough scaling of $t \sim r / v$ (where $r$ is the size of the spatial variabilities and $v$ is the corresponding propagation speed) seems to be applied, the ultimate understanding of the physical processes involved in the variabilities could possibly be achieved only through detailed MHD modeling, including simulations that are beyond the scope of this paper.

CYH is supported by the National Research Foundation of Korea through grants 2014R1A1A2058590 and 2016R1A5A 1013277. JL is supported by the BK21 plus program and 2016R1A5A1013277. AKHK is supported by the Ministry of Science and Technology of Taiwan through grants 103-2628M-007-003-MY3 and 105-2112-M-007-033-MY2. PHT is supported by the One Hundred Talents Program of Sun YatSen University. JT is supported by the NSFC grants of China under 11573010. KSC is supported by a 2014 GRF grant of the Hong Kong government under HKU 17300814P. DR is supported by the National Research Foundation of Korea through grant 2016R1A5A1013277. 


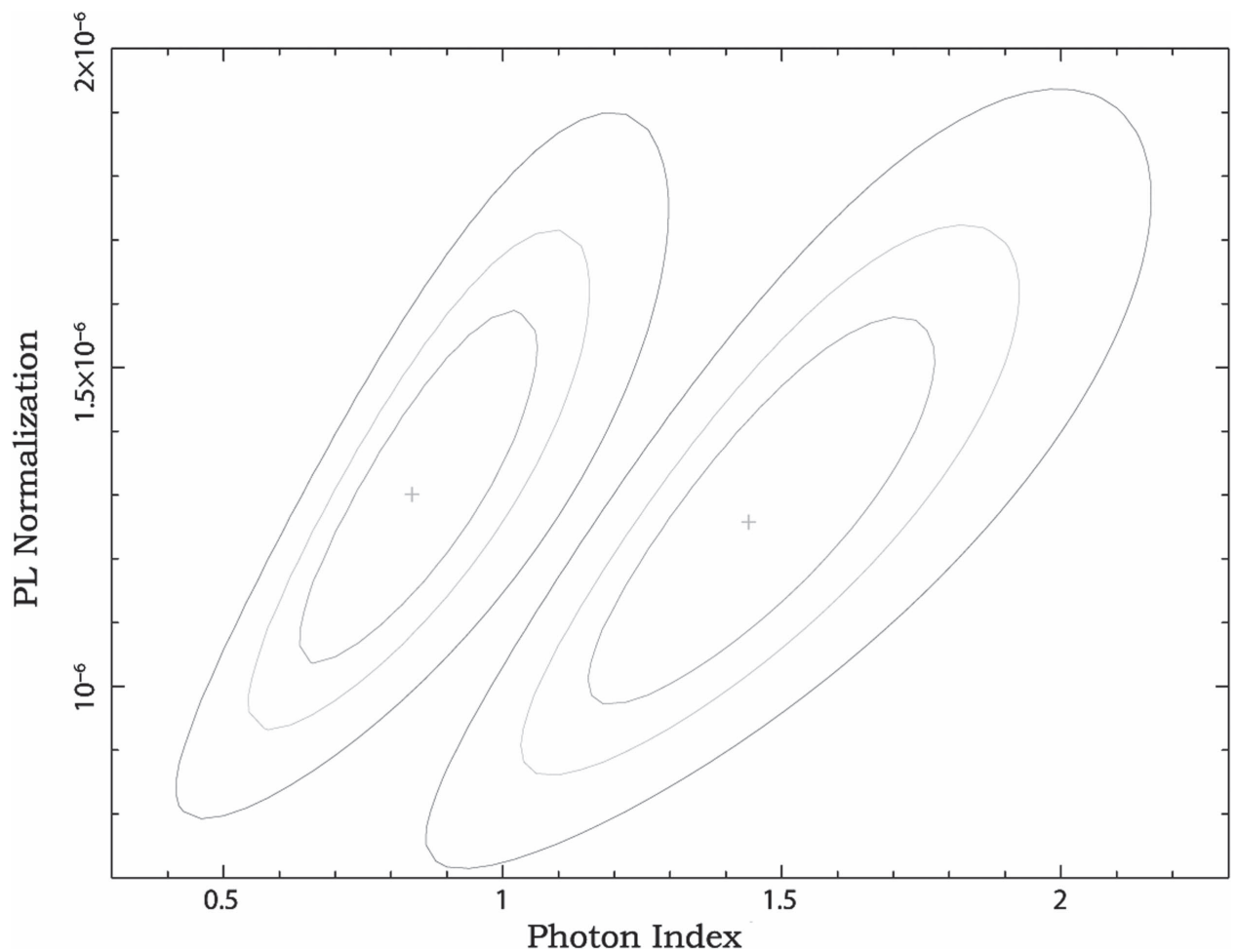

Figure 14. The $1 \sigma, 2 \sigma$, and $3 \sigma$ confidence contours for the parameters of the front part (right contours) and rear part (left contours) of the outer southern tail derived in the period of Epochs 4-8. Spectral hardening along the tail is noticed in this period.

\section{ORCID iDs}

C. Y. Hui $\odot$ https://orcid.org/0000-0003-1753-1660

P. H. T. Tam (1) https://orcid.org/0000-0002-1262-7375

J. Takata (1) https://orcid.org/0000-0002-8731-0129

Dongsu Ryu (1) https://orcid.org/0000-0002-5455-2957

\section{References}

Arons, J. 2012, SSRv, 173, 341

Begelman, M. C., Rees, M. J., \& Blandford, R. D. 1979, Natur, 279, 770

Caraveo, P. A., Bignami, G. F., De Luca, A., et al. 2003, Sci, 301, 1345

De Luca, A., Caraveo, P. A., Mereghetti, S., Negroni, M., \& Bignami, G. F. 2005, ApJ, 625, 1051

Faherty, J., Walter, F. M., \& Anderson, J. 2007, Ap\&SS, 308, 225

Gaensler, B. M., \& Slane, P. O. 2006, ARA\&A, 44, 17

Gold, T. 1969, Natur, 221, 25

Halpern, J. P., \& Wang, F. Y.-H. 1997, ApJ, 477, 905

Li, T.-P., \& Ma, Y.-Q. 1983, ApJ, 272, 317
Manchester, R. N., Hobbs, G. B., Teoh, A., \& Hobbs, M. 2005, ApJ, 129, 1993 McBride, J., \& McCourt, M. 2014, MNRAS, 442, 838

Mizuno, Y., Hardee, P. E., \& Nishikawa, K.-I. 2014, ApJ, 784, 167

Mori, K., Gotthelf, E. V., Dufour, F., et al. 2014, ApJ, 793, 88

Mori, K., et al. 2004, in IAU Symp. 218, Young Neutron Stars and Their Environments, ed. F. Camilo \& B. M. Gaensler (San Francisco, CA: ASP), 181 Noutsos, A., Kramer, M., Carr, P., et al. 2012, MNRAS, 423, 2736

Pavlov, G. G., Bhattacharyya, S., \& Zavlin, V. E. 2010, ApJ, 715, 66 Pavlov, G. G., Sanwal, D., \& Zavlin, V. E. 2006, ApJ, 643, 1146

Pavlov, G. G., Teter, M. A., Kargaltsev, O., \& Sanwal, D 2003, ApJ, 591, 1157

Porth, O., Buehler, R., Olmi, B., et al. 2017, SSRv, 207, 137

Posselt, B., Pavlov, G. G., Slane, P. O., et al. 2017, ApJ, 835, 66

Rees, M. J., \& Gunn, J. E. 1974, MNRAS, 167, 1

Sanwal, D., Pavlov, G. G., \& Zavlin, V. E. 2004, BAAS, 36, 923

Singh, C. B., Mizuno, Y., \& de Gouveia Dal Pino, E. M. 2016, ApJ, 824, 48 Spruit, H., \& Phinney, E. S. 1998, Natur, 393, 139

Srivastava, A. K., Shetye, J., Murawski, K., et al. 2017, NatSR, 7, 43147

Szente, J., Toth, G., Manchester, W. B., IV, et al. 2017, ApJ, 834, 123

Weisskopf, M. C., Hester, J. J., Tennant, A. F., et al. 2000, ApJL, 538, L81

Zhang, L., \& Cheng, K. S. 2001, MNRAS, 320, 477 